\title{
Acknowledgement to Reviewers of Molecules in 2016
}

\author{
Molecules Editorial Office \\ MDPI AG, St. Alban-Anlage 66, 4052 Basel, Switzerland; molecules@mdpi.com \\ Published: 11 January 2017
}

The editors of Molecules would like to express their sincere gratitude to the following reviewers for assessing manuscripts in 2016.

We greatly appreciate the contribution of expert reviewers, which is crucial to the journal's editorial process. We aim to recognize reviewer contributions through several mechanisms, of which the annual publication of reviewer names is one. Reviewers receive a voucher entitling them to a discount on their next MDPI publication and can download a certificate of recognition directly from our submission system. Additionally, reviewers can sign up to the service Publons (https://publons.com) to receive recognition. Of course, in these initiatives we are careful not to compromise reviewer confidentiality. Many reviewers see their work as a voluntary and often unseen part of their role as researchers. We are grateful to the time reviewers donate to our journals and the contribution they make.

If you are interested in becoming a reviewer for Molecules, see the link at the bottom of the webpage http://www.mdpi.com/reviewers.

The following reviewed for Molecules in 2016:

Abarbri, Mohamed

Abdi, Salahadin

Abe, Sumiko

Abenavoli, Ludovico

Abitbol, Tiffany

Abourashed, Ehab

Abraham, E. M.

Abriata, Luciano A.

Abuelo, Ángel

Abu-Reidah, Ibrahim M.

Acero, $\mathrm{N}$.

Ackerman, H. C.

Acosta, Edgar

Ademiluyi,

Adedayo Oluwaseun

Afaq, Farrukh

Afonso, Clélia N.

Agerbirk, Niels

Agrawal, Prashansa

Agudelo-Romero, Patricia

Aguilar, Alfredo

Ahmad, Nihal
Ahmed, Nisar

Ahn, Mok-Ryeon

Ahn, Sangdoo

Aiello, Francesca

Aires, Alfredo

Akhavan, Omid

Akhmedov, Novruz G.

Akitsu, Takashiro

Alabugin, I.

Alauddin, Mian M

Albini, Angelo

Al-Dujaili, Emad

Alexandra M. M., Antunes

Alexandre-Gouabau,

Marie-cécile F.

Alexiou, Christoph

Al-Horani, Rami

Alikhani, Esmail

Allais, Florent

Allen, Jeremy

Aller, Patricio

Allwood, Daniel M.
Almajano, María Pilar

Almansa, Carmen

Almerico, Anna Maria

Altomare, Cosimo

Alvarenga, Elson

Álvarez, Mercedes

Alvarez Ortí, Manuel

Alvarez-Lorenzo, Carmen

Alvarez-Suarez, Josè Miguel

Alves, Eliana

Alway, Stephen E.

Amarowicz, Ryszard

Ambrogi, Valeria

Amiche, Mohamed

Amorós, Asunción

Amouri, Hani

Amslinger, Sabine

An, Hyun Joo

An, Jia

Anas, Andrea Roxanne

Andersen, Grethe Neumann

Anderson, Anne J. 
Anderson, Robin C.

Andrade, Paula

Andreadou, Ioanna

Andreana, Peter

Andreoli, Mirko

Andreoli, Roberta

Andrianasolo, Eric

Anes, Elsa

Angelini, Paola

Angelino, Donato

Angelone, Tommaso

Angulo, Javier

Anna, Vallverdú-Queralt

Anne, Laurençon

Annese, Cosimo

Antal, Diana Simona

Antalick, Guillaume

Antoine, Marie-Hélène

Anton, Steve

Antonchick, Andrey

Antoniotti, Sylvain

Antunes, Lusânia

Anzai, Jun-ichi

Aojula, Harmesh S.

Aoki, Wataru

Aoki, Yoshitsugu

Apone, Fabio

Appendino, Giovanni

Aprea, Eugenio

Aragones, Gerard

Arai, Midori A.

Arai, Takayoshi

Aranda, Agustín

Araniti, Fabrizio

Argyriou, Anagnostis

Argyropoulou, Aikaterini

Arias, Renée S.

Ariga, Katsuhiko

Armanini, Decio

Armenta, Sergio

Armitage, Ruth Ann

Arnason, John

Arnhold, Juergen
Arnold, Eva

Arrick, Denise

Arru, Laura

Arterburn, Jeffrey B.

Arulselvan, Palasamy

Arvapally, Ravi Kumar

Asai, Akira

Asai, Daisuke

Asai, Teigo

Ashida, Hitoshi

Askalan, Rand

Asuero, Agustín G.

Ata, Athar

Atkins, William M.

Atluri, Venkata S.R.

Attard, Thomas

Atzori, Luigi

Aubert, Emmanuel

Avarvari, Narcis

Avato, Pinarosa

Averbeck, Dietrich

Av-Gay, Yossef

Awual, Md. Rabiul

Azémaa, Joëlle

Azerad, Robert

Aznauryan, Mikayel

Azqueta, Amaya

Azuma, Kazuo

Azzimonti, Barbara

Babu, Dinesh

Bach, Horacio

Bachawala, Praveen

Back, Kyoungwhen

Bácskay, Ildikó

Bączek, Tomasz

Badu, Shyam R.

Baes, Myriam

Baeza, Alejandro

Bafor, Enitome

Bai, Gang

Bai, Shi-Qiang

Bai, Shuhua

Bailey-Kellogg, Chris
Bajerska, Joanna

Bajpai, Richa

Bakare, Oladapo

Bakker, FT (Freek)

Balawender, Robert

Baláž, Matej

Balemba, Onesmo B.

Bálint, Erika

Ballerini, Clara

Ballester, Pedro J.

Ballesteros, Antonio

Ban, Hyun Seung

Banach, Marcin

Banc, Amelie

Banc, Amélie

Banerjee, Aditi

Banerjee, Hirendra Nath

Banfalvi, Zsofia

Bankova, Vassya

Bannister, Thomas

Bannwarth, Markus B.

Banskota, Arjun H.

Banti, Christina N.

Bao, Jie

Bao, Jinku

Bao, Yongping

Baran, Timothy M.

Barba, Francisco

Barbieri, Giuseppe

Barbosa, Oveimar

Barbosa Pereira, Letricia

Barcelos, Gustavo

Barclay, Thomas

Barone, Justin

Barraja, Paola

Barrajón-Catalán, Enrique

Barraud, Nicolas

Barreira, João C.M.

Barreiro, Sonia Losada

Barrio, Pablo

Barron, Andrew R.

Barroso-Bogeat, Adrián

Bartolini, Manuela 


\begin{tabular}{|c|c|c|}
\hline Bartolomé, Begoña & Beppu, Fumiaki & Bills, Gerald \\
\hline Bartolotti, Massimo & Beretta, Giangiacomo & Bimbo, Luis M. \\
\hline Basco, Leonardo K. & Bergbreiter, David E & Birch, John \\
\hline Basiak, Ewelina & Bergens, Steven & Bishayee, Anupam \\
\hline Basini, Giuseppina & Berger, Ralf G & Bishop, Anthony C. \\
\hline Basso, Andrea & Bergès, Thierry & Bishop-Hurley, Sharon \\
\hline Basso, Daniela & Berhow, Mark & Bisignano, Giuseppe \\
\hline Battino, Maurizio & Berlicki, Łukasz & Bisti, Silvia \\
\hline Baudoux, Jérôme & Berlin, K. Darrell & Bisztray, Gyoergy D. \\
\hline Bauer, Tomasz & Bernal, Claudia & Bjørndal, Bodil \\
\hline Baum, Christel & Bernard, Denzil & Blair, Jessica \\
\hline Bavaresco, Luigi & Bernardi, Luca & Blais, Anne \\
\hline Bayer, Ilker S. & Bernhardt, Harold S. & Blakely, Randy \\
\hline Bayry, Jagadeesh & Bernhoft, Aksel & Blanco, Rosa M. \\
\hline Bazaka, Kateryna & Bernini, Roberta & Błaszczak-Świątkiewicz, \\
\hline Bazylińska, Urszula & Berrie, Cindy & Katarzyna \\
\hline Beall, Gary W. & Berta, Graziella & Błażewska, Katarzyna M. \\
\hline Beaudoin, Claude & Berti, Federico & Blazquez, M. Amparo \\
\hline Beaudry, Chris & Bertinaria, Massimo & Bloem, Elke \\
\hline Beauvais, Anne & Bertolini, Joseph & Boa, Andrew N. \\
\hline Beberok, Artur & Bérubé, Gervais & Bobe, Gerd \\
\hline Beck, Andreas & Besada, Cristina & Boddy, Christopher N. \\
\hline Bedini, Emiliano & Besalú, Emili & Boechat, Nubia \\
\hline Bednarczyk-Cwynar, Barbara & Besson, Thierry & Boege, Fritz \\
\hline Bednarska, Katarzyna & Bethanis, Kostas & Boeglin, Alex \\
\hline Bednarski, Patrick & Beuerle, Florian & Boga, Carla \\
\hline Beier, Ross C. & Bezdetnaya, Lina & Böhm, Volker \\
\hline Beld, Joris & Bezirtzoglou, Eugenia & Bohn, Torsten \\
\hline Belfield, Kevin D. & Bhagavathy, & Boitel-Conti, Michèle \\
\hline Belfrage, Anna Karin & Ganga Viswanathan & Bojarski, Andrzej \\
\hline Belide, Srinivas & Bhattacharya, Chandrabali & Bolibok-Brągoszewska, \\
\hline Belitsky, Jason M. & Bhosale, Sheshanath & Hanna \\
\hline Bell, Thomas W. & Bhutoria, Savita & Bolivar, Juan \\
\hline Bellés, José María & Bi, Kaishun & Bolm, Carsten \\
\hline Bello, Nicholas T. & Biagini, Giuseppe & Bolognesi, Andrea \\
\hline Bełtowski, Jerzy & Bianchi, Antonio & Bolotov, Leonid \\
\hline Benaglia, Maurizio & Bianco, Armando Doriano & Bondon, Arnaud \\
\hline Bencharit, Sompop & Biava, Mariangela & Bondonno, Catherine \\
\hline Benelli, Giovanni & Biavatti, Maique W. & Boni, Adriano \\
\hline Benito, José Manuel & Biddle, Adrian & Bonifácio, Vasco \\
\hline Benito, Santiago & Biedermann, David & Bonilla, C. E. P. \\
\hline Benson, Heather & Bijak, Michał & Bonnet, Veronique \\
\hline Benzeroual, Kenza E. & Billard, Thierry & Boo, Yong Chool \\
\hline
\end{tabular}




\begin{tabular}{|c|}
\hline Borbas, K. Eszter \\
\hline Bordes, Patricia \\
\hline Borges, Anabela \\
\hline Borochov-Neori, Hamutal \\
\hline Borondo, Florentino \\
\hline Borowska, Marta \\
\hline Borsani Cambón, Julio Omar \\
\hline Boryczka, Stanisław \\
\hline Bosch, Joaquim \\
\hline Botelho, Goreti \\
\hline Botre, Francesco \\
\hline Boulares, Hamid \\
\hline Bouquillon, Sandrine \\
\hline Bour, Petr \\
\hline Bousbaa, Hassan \\
\hline Bouvier, Guillaume \\
\hline Bowden, Bruce \\
\hline Bowling, Lee \\
\hline Bowman, Michael \\
\hline Boyd, Benjamin \\
\hline Boyle, Joseph J. \\
\hline Bracher, Franz \\
\hline Brachet, Etienne \\
\hline Braga, Susana \\
\hline Brain, Susan D. \\
\hline Bramley, Peter \\
\hline Branca, Carmen \\
\hline Branca, Ferdinando \\
\hline Branchini, Alessio \\
\hline Brand, D. Jaco \\
\hline Brandão, Maria Das Graças \\
\hline Bravo, Laura \\
\hline Bravo López, Susana B. \\
\hline Breaker, Ronald \\
\hline Brecker, Lothar \\
\hline Breinbauer, Rolf \\
\hline Breitenfeld Granadeiro, Luiza \\
\hline Bremner, John B. \\
\hline Brennan, Marian \\
\hline Breydo, Leonid \\
\hline Briceño, Gabriela \\
\hline Brindisi, Margherita \\
\hline Broderick, Tom L. \\
\hline
\end{tabular}

Broisat, Alexis

Brooks, Amanda E.

Brouard, Danny

Brown, Darren

Brown, Lindsay

Brück, Thomas

Bruckner, Christian

Bruin, Bas De

Brullo, Chiara

Brun, Reto

Brunetti, Cecilia

Bruni, Giovanna

Bruni, Renato

Brunklaus, Gunther

Bruns, Nico

Brusotti, Gloria

Brycki, Bogumił

Brzezinski, Ryszard

Bučar, Dejan-Krešimir

Buchowicz, W.

Buckley, M.

Buddle, Bryce

Budzisz, Elzbieta

Buemi, Maria Rosa

Bugaut, Xavier

Bunce, Richard A.

Bunker, Alex

Bunz, Uwe H. F.

Buonanno, Manuela

Burgos Hernández, Armando

Burley, Jonathan C.

Buron, Frédéric

Burow, Meike

Burris, Kellie P.

Burton-Freeman, Britt

Burton-Freeman, M.

Busconi, Matteo

Bushman, Shaun

Businaro, Rita

Butini, Stefania

Buxton, Denis

Bystrowska, Beata

Caballero, Julio
Cabedo, Nuria

Cabral, Célia

Cabral, Horacio

Cabral, Jaydee

Cadwallader, Keith

Caelles Franch, Carmen

Caffarelli, Carlo

Caffrey, Patrick

Cafiero, Mauricio

Cai, Lisheng

Cai, Xianghai

Calderone, Vincenzo

Calhelha, Ricardo C.

Calogeropoulou, Theodora

Calvete, Juan

Calvete, Mario

Camacho, M. Encarnación

Camara, Amadou K.S.

Cámara-Martos, Fernando

Camarasa, María-José

Camele, Ippolito

Camins, Antoni

Cammas-Marion, Sandrine

Campana, Raffaella

Campbell, Christopher

Campbell, Lee

Campos Rosa, Joaquín

Canas, Sara

Candiani, Gabriele

Canela, Dr. Ramon

Canini, Antonella

Cannazza, Giuseppe

Cao, Bin

Cao, Bo

Cao, Wei

Cao, Zexing

Capasso, Raffaele

Capece, Angela

Capriati, Vito

Carabineiro, Sónia A. C.

Caramori, Stefano

Caramori, Giovanni F.

Carbone, Anna 


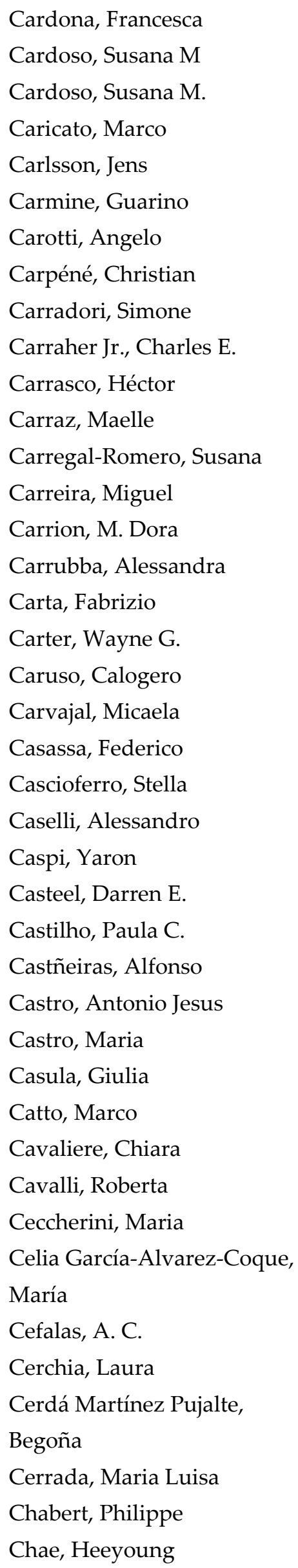

Chai, Yifeng

Chaimbault, Patrick

Chalker, Justin M.

Challinor, Victoria L.

Chambers, Karen

Chamulitrat, Walee

Chan, Ben C. L.

Chan, Tak Hang

Chan, Thomas

Chan, Vincent

Chand, Subhash

Chang, Chi K.

Chang, Chih-Jui

Chang, Chih-Wei

Chang, Chih-Yu

Chang, Chiou Ling

Chang, Fang-Rong

Chang, Hsueh-Wei

Chang, Hyeun Wook

Chang, Li-Ching

Chang, Long-Sen

Chang, Sue-joan

Chang, Yao-Feng

Chang, Yuan-Shiun

Chao, Louis Kuoping

Chao, Ming-Wei

Chao, Pi-Yu

Chao, Yanjie

Chapoval, Svetlana P.

Chappell, Joseph

Charles, Marie Thérèse

Chatel, Grégory

Chatterjee, Jhinuk

Chaturvedi, Pankaj

Chaves-Lopez, Clemencia

Chavez, Ferman

Chazot, Paul L.

Che, Fang-Sik

Checa, Antonio

Chefetz, Benny

Chemat, Farid

Chen, Cheng-Lung

Chen, Chung-Yi
Chen, Chun-Jung

Chen, Chun-Long

Chen, Daiqin

Chen, Dajun

Chen, Fen-Er

Chen, Gao

Chen, Guibing

Chen, Gwo-Shing

Chen, Heru

Chen, Hsin-Chun

Chen, Huaiqiong

Chen, Huimei

Chen, Hui-yin

Chen, Jian-Zhong

Chen, Ji-hua

Chen, Jinhui

Chen, Jinquan

Chen, Li

Chen, Menglin

Chen, Miaw-Ling

Chen, Min-Huey

Chen, Mohan

Chen, Po-Yuan

Chen, Qi

Chen, Ruei-ming

Chen, Shilin

Chen, Shiu-Nan

Chen, Shuai

Chen, Tianbao

Chen, Tong

Chen, Xiaolan

Chen, Xin

Chen, Yingchun

Chen, Yu-Kuo

Chen, Yun-Wen

Chen, Zheng (Jake)

Cheng, Heung-Chin

Cheng, Jing-Jy

Cheng, Jizhong

Cheng, Juei-Tang

Cheng, Qing-Qing

Cheng, S. S.

Cheng, Shi-yie 


\begin{tabular}{|c|}
\hline Cheng, Sun \\
\hline Cheng, Xingguo \\
\hline Cheng, Yi-Xiang \\
\hline Cheng, Yong \\
\hline Cheng, Yu-Wen \\
\hline Cheng, Zhiqiang \\
\hline Chenia, Hafizah \\
\hline Cherkaoui-Malki, Mustapha \\
\hline Chern, Ming-Kai \\
\hline Cherniack, E. Paul \\
\hline Chetcuti, Michael J. \\
\hline Cheung, Randy Chi Fai \\
\hline Cheung, Wing-Hoi \\
\hline Chew, Eng-Hui \\
\hline Chi, Chun Y. \\
\hline Chi, Yun \\
\hline Chia, Win-Long \\
\hline Chiang, Cheng-Yang \\
\hline Chiang, Hsiu-Mei \\
\hline Chiang, Long Y. \\
\hline Chiang, Shen-Shih \\
\hline Chiang, Tai-an \\
\hline Chiang, Wen-Dee \\
\hline Chiarelli, Laurent \\
\hline Chi-Feng, Hung \\
\hline Chilmonczyk, Zdzisław \\
\hline Chimento, Adele \\
\hline Chin, Young-Won \\
\hline Chinchilla, Rafael \\
\hline Chinnici, Fabio \\
\hline Chiocchia, Gilles \\
\hline Chiou, Shih-hwa \\
\hline Chiou, Wen-fei \\
\hline Chisholm, John D. \\
\hline Chitwood, David \\
\hline Chiu, Tien-Lung \\
\hline Chiu, Tsai-Hsin \\
\hline Chiva-Blanch, Gemma \\
\hline Chivers, Ian H. \\
\hline Chlichlia, Katerina \\
\hline Cho, Chunghee \\
\hline Cho, Dong-Woo \\
\hline Cho, Hyunah \\
\hline
\end{tabular}

Cho, Man-Ho

Cho, Namjoon

Cho, Somi Kim

Chobot, Vladimir

Choi, Eun-Mi

Choi, Guang J.

Choi, Hyeonson

Choi, Hyung-Kyoon

Choi, Ki Choon

Choi, Seok Ki

Choi, Seokheun

Choi, Young Hae

Choi, Young Hee

Choi, Yung Hyun

Cholewinski, Grzegorz

Choshi, Tominari

Chou, C. James

Chou, Timothy Y.

Choy, Jin-HO

Chrissanthopoulos,

Athanassios

Christensen, Jørn B.

Christensen, Lars

Chrobok, A.

Chronis, Nikos

Chruszcz, Maksymilian

Chrzanowski, Łukasz

Chu, Dafeng

Chu, Jiaxiang

Chu, Shidong

Chua, Mei-Sze

Chuang, Li-yeh

Chuang, Show-mei

Chuang, Ta-hsien

Chueh, Pin Ju

Chulvi, Katherine

Chun, Byung-soo

Chung, Donghoon

Chung, Jong-Wook

Chung, Sang Jeon

Chung, Soonkyu

Chung, Yoon-Sok

Churchill, Mair E. A.
Chwalbe, Carl H. S.

Chyau, Charng-Cherng

Cichero, Elena

Cidade, Honorina

Cieśla, Łukasz

Cimanga, Kanyanga

Ciofu, Oana

Cirillo, Giuseppe

Cirrincione, Girolamo

Cirvilleri, Gabriella

Cisnetti, Federico

Citores, Lucia

Ciulli, Alessio

Ciurli, Stefano

Civra, Andrea

Clark, Daniel A.

Clements, Carol

Clift, Martin

Cobbold, Simon

Cocetta, Giacomo

Cock, Ian Edwin

Coelho, José A.

Cognasse, Fabrice

Cohen, Haim

Coley, Helen M.

Coll, Josep

Collins, Maurice

Collins, Stuart G.

Collis, Gavin E.

Colombo, Claudio

Colquhoun, Thomas A.

Columbano, Amedeo

Comai, Stefano

Conceição, Katia

Consonni, Roberto

Constantino, Valeria

Conti, Barbara

Conti, Pio

Contreras, Angela

Contreras, Lucila Garcia

Contreras, María Del Mar

Cooper, Arthur

Corchete, Purificacion 
Corcione, Carola Esposito

Cordoba-Diaz, D.

Corella, Dolores

Corke, Harold

Cormode, David P.

Cornaglia, M.

Corral, Inés

Correa-Basurto, Jose

Correia Soeiro,

Maria De Nazaré

Correia-de-Sá, Paulo

Cortes Corberán, Vicente

Costa, Elisabete V.

Costa, Maria Do Céu

Gonçalves Da

Costa, Rosaria

Costa Pessela João, Benevides

Costantini, Maria

Costantino, Ferdinando

Costantino, Valeria

Costas, Miquel

Couce, María D.

Couch, Robin

Cournia, Zoe

Cowan, Tina

Cox, Jane

Cox, Russell J.

Cozzolino, Daniel

Crabtree, Robert H.

Cranfield, Charles

Crean, Daniel

Crecchio, Carmine

Crespo, Margarita

Crinò, Paola

Crispin, Matthew C.

Crisponi, Guido

Cruse, Richard

Csámpai, Antal

Csiszár, Agnes

Csuk, Rene

Cuculovic, Milos

Cuellar, Mauricio

Cui, Zining
Cullis, Christopher

Cunha, Mário

Curti, Claudio

Custódio, Luísa

Cutignano, Adele

Cutrignelli, Annalisa

Czechowski, Tomasz

Czerwinska, Monika

Da Costa, Gonçalo Gamboa

Da Motta, M.

Da Silva, Edson Roberto

Da Silva, Felipe M. A.

D'abrosca, Brigida

Dabrowski, Janusz

D'accolti, Lucia

Dacheux, Jean-Louis

Dai, Haofu

Dai, Yumin

Dai, Yun

Dai, Zhanwu

Daina, Antoine

Dal Monte, Massimo

Dalhoff, Axel

Dall'Acqua, Stefano

Daly, Seth

Da'na, Enshirah

Dang, Jing-Shuang

Daniels-Wells, Tracy R.

Danneels, Ellen L.

Dar, Mohammad Ibrahim

Dardonville, Christophe

Das, Deepankar

Das, Viswanath

Dasari, Ramesh

Dasgupta, Piyali

Dasgupta, Sayani

Daugaard, Anders Egede

D'Auria, John

D'Auria, Maria Valeria

D'Auria, Maurizio

Dauwe, Rebecca

Daverey, Amita

Davies, Gemma-Louise
Davis, Burt

Davis, Rohan

Dawadi, Surendra

De Aberasturi,

Dorleta Jiménez

De Almeida,

Martha Elisa Ferreira

De Almeida Coelho De Sousa,

Maria João

De Aza, Piedad N.

De Beer, Dalene

De Caro, Viviana

De Castro, Solange

De Corato, Ugo

De Dea Lindner, Juliano

De Falco, Enrica

De Feo, Vincenzo

De Figueiredo, Marcia

De Freitas, Victor

De Freitas Gauze, Gisele

De Geest, Bart

De Graaf, Coen

De Juan, Anna

De Kimpe, $\mathrm{N}$

De La Hoz, Antonio

De La Monte, Suzanne

De La Torre,

Maria Del Carmen

De Luca, Stefania

De Melo Barbosa Dekker,

Aneli

De Mendonça, Dina I. M. D.

De Menezes, Irwin R. A.

De Pauw, Edwin

De Rijke, Eva

De Vendittis, Emmanuele

De Vries, Mattanjah

Deda, Massimo La

Dehaen, Wim

Dekanski, Dragana

Dekker, Frank

Del Bonis-O'Donnell,

Jackson Travis 


Del Mercato, Loretta
Del Pozo Losada, Carlos
Delattre, Cédric
Delgadillo, Adriana San Miguel
Della Ca, Nicola
DellaGreca, Marina
Delpino, Antoni
Demchenko, Alexei

Demirci, Prof. Dr. Fatih

Den Hertog, Jeroen

Denat, Franck

Deng, Jianjun

Deng, Jun

Deng, Lih-Wen

Dentino, Andrew R.

Derat, Etienne

Desimone, Martin Federico

Dessy, Chantal

Deurwaerdère, Philippe

Devamani, Titu

Devriendt, Bert

Dey, Priyankar

Dhakshinamoorthy,

Amarajothi

Dhanasekaran, Danny N.

Di Luca, Mariagrazia

Di Maro, Antimo

Di Martino, Piera

Di Santo, Roberto

Di Serio, Martino

Di Somma, Ilaria

Diana, Patrizia

Diana, Patrizia

Díaz, José Fernando

Diederich, Marc

Díez, David

Dijkhuizen, Lubbert

Dikalov, Sergey

Ding, Chunbang

Ding, Junqiao

Dinh, Dzung H.

Diniz, Carmen

Dipali, Sharma
Dixon, David A.

Djabali, Karima

Djordjevic, Snezana

Dmitriev, Ruslan

Dobrev, Dobromir

Dolzhenko, Anton V.

Domashevskiy, Artem V.

Domcke, Wolfgang

Dominguez, Herminia

Domínguez-Álvarez, Enrique

Donalisio, Manuela

Dong, F.

Dong, Mingdong

Dongari-Bagtzoglou, Anna

Donno, Dario

Dorta, Reto

dos Santos, Jean Leandro

Dosio, Franco

Dou, Q. Ping

Douroumis, Dionysios

Dovinova, Ima

Dowden, James

Drabowicz, Jozef

Drabowicz, Józef

Dragonetti, Claudia

Draijer, Richard

Drasar, Pavel

Du, Haifeng

Du, Shushan

Duan, Jinao

Duan, Xiaohui

Duarte, Ana Rita C.

Duarte, Fátima

Duarte Campos, Daniela Filipa

Duba, Kurabachew

Dubey, Ramin

Dubois, Joëlle

Dubois, Marc

Ducki, Sylivie

Dudás, József

Dudding, Travis

Düfer, Martina

Duggan, Brendan
Dughera, Stefano

Dugo, Giacomo

Duh, Chang-Yih

Duke, Stephen O.

Dumas, Françoise

Dumont, Elise

Dumur, Frédéric

Dunietz, Barry D.

Duong, Hai M.

Dupont, Daniel M.

Duran Lobato, Matilde

Durand, Jean Olivier

Durazzo, Alessandra

Dusselier, Michiel

Dust, Julian M.

Duval, Raphael E.

Dzantiev, Boris

Dziadek, Jarosław

Dziedzic, Arkadiusz

Dziubla, Thomas

Dzyuba, Sergei

Easmon, Johnny

Ebrahim, Weaam

Echevarria, Aurea

Ecker, Gerhard F.

Edokpayi, Joshua

Efferth, Thomas

Efimia, Hatzidimitriou

Eguchi, Yutaka

Eiden, Lee E.

Ejima, Hirotaka

Ekuni, Daisuke

El-Aneed, Anas

Eldeeb, Mohamed

Eleftherianos, Ioannis

Elisabetta, Casalino

El-Kadi, Ayman OS

El-Safty, Sherif A.

El-Seedi, Hesham R.

Elsinghorst, Paul W.

Emanuelsson, Emma

Emmott, Edward

Empting, Martin 
Engels, Joachim

Engle, Keary M.

Eparvier, Véronique

Epifano, Francesco

Epstein, Alan

Erdélyi, Máté

Eriksson, Leif A.

Eritja, Ramon

Ertl, Peter

Erxleben, Andrea

Esatbeyoglu, Tuba

Escalante García, Jaime

Escudier, Jean-Marc

Escuredo, Olga

Espinosa Aguirre, Javier

Espinosa-Diez, Cristina

Esposito, Sergio

Esteban, Maria Angeles

Estevez, Ramón J.

Estévez-Braun, Ana

Estevinho, Leticia

Etchenique, Roberto

Eto, Bruno

Euaggelos, Gikas

Evanno, Laurent

Evans, Paul

Everts, Bart

Exarchou, Vassiliki

Fábián, László

Fabiano-Tixier, Anne-Sylvie

Fabre, Nicolas

Faccio, Greta

Faggio, Caterina

Fakis, Mihalis

Famiani, F.

Fan, Hong

Fan, Qinghua

Fanali, Chiara

Fañanas, Francisco J.

Fanfrlík, Jindřich

Fang, Bai

Fang, Marong

Fanizzi, Francesco P.
Fanning, Kent J.

Fant, Jeremie B.

Faraji, Shirin

Farantos, Stavros C.

Faria, Jorge M.S.

Farinati, Fabio

Farris, Stefano

Fasani, Elisa

Faure, Chrystel

Faure, Sébastien

Faustino, Maria Amparo

Fávero, Priscila Pereira

Fedorova, Maria

Fedrizzi, Bruno

Feliers, Denis

Felinger, Attila

Felipe Farias, Davi

Felpin, François-xavier

Feng, Quanyou

Feng, Weisheng

Feng, Wen-hai

Feng, Yangbo

Feng, Yibin

Feng, Yong-Lai

Feng, Yu

Fennell, Timothy R.

Feo, Francesco

Fernandes, Eduarda

Fernandes, Pedro

Fernandes, Susana C.M.

Fernandes-Ferreira, Manuel

Fernandez, Esther

Fernández, Francisco J.

Fernández, Inmaculada

Fernández, José J.

Fernández, Rosario

Fernandez Megia, Eduardo

Fernandez-Garcia, Marta

Fernandez-Lafuente, Roberto

Fernández-Recio, Juan

Feron, Krishna

Ferrari, Erika

Ferraris, Dana V
Ferraz, Ricardo

Ferré-D'Amaré, Adrian R.

Ferrero, Franco

Ferretti, Valeria

Figadère, Bruno

Figueira, Etelvina

Figueiral, Maria Helena

Figueiredo, Ana Cristina

Figuerola, Albert

Filarowski, Aleksander

Filipovic, Milos R.

Filosa, Rosanna

Fiocco, Daniela

Fisar, Zdenek

Fisher, Jed F.

Fissell, William

Fitó, Montserrat

Fitton, Helen

Fleischer, Ivana

Fleming, Ingrid

Fletcher, James

Flood, Amar

Floreancig, Paul E.

Florian, Pitterl

Fochi, Mariafrancesca

Fons, Françoise

Foo, Eloise

Forlani, Giuseppe

Forsyth, Christopher B.

Fort, Francesca

Fortes, Zuleica

Fortier, Skye

Fossa, Paola

Fossen, Torgils

Fossum, Magdalena

Foster, Brian C

Fosu-Mensah, Nelly

Fouassier, Laura

Foubelo, Francisco

Foulongne-Oriol, Marie

Fourmy, Daniel

Fox, Glenn

Fraga, Marco A. 
Fraile, Alberto

Fraile, José M.

Franca, Eduardo F

Franceschinis, E.

Francesco, Masoero

Francioso, Antonio

Frank, Saša

Franzke, Claus-Werner

Freire, Félix

Freitas, Marisa

Fribley, Andrew M.

Froeyen, Matheus

Frolova, Liliya

Frosini, Maria

Fruhmann, Philipp

$\mathrm{Fu}$, Gang

$\mathrm{Fu}$, Junjie

$\mathrm{Fu}$, Qiang

Fuchs, Hendrik

Fudala, Rafal

Fujihara, Tetsuaki

Fujii, Kotaro

Fujimori, Ko

Fujimoto, Yukari

Fujita, Masahiro

Fujita, Mikako

Fujiwara, Ryoichi

Fukuda, Takeshi

Fukushima, Atsushi

Fukushima, Masami

Fuller, Amelia A.

G. Lago, João Henrique

Gadotti, Vinicius M

Gai, Francesco

Galanis, Alex

Galan-Vidal, Carlos Andres

Galdiero, Massimiliano

Galeazzi, Roberta

Gales, Luís

Gallagher, John F.

Gallego, Sergi

Gallo, Antonio

Gallo, Carmela
Gallo, Giuseppe

Galvez, Julio

Galvez, Maria Elena

Gan, Chee-Yuen

Gan, Renyou

Ganesan, A.

Ganguly, Anutosh

Gao, Baoxiang

Gao, Jie

Gao, Jinming

Gao, Li Qian

Gao, Song

Gao, Xue

Garaj, Vladimír

Garavelli, Marco

Garbers, Christoph

Garcia, Diego

Garcia, Jordi

García, Tania

García Csákÿ, Aurelio

García-Antón, Jordi

Garcia-Gonzalez, Carlos

García-Ortega, Lucía

Garcia-Sosa, Alfonso T.

García-Verdugo, Eduardo

García-Villalón, Angel Luis

Gard, Paul

Gardossi, Lucia

Garg, Akhil

Garg, Uttam

Garneau-Tsodikova, Sylvie

Garrido, Daniel Valero

Garrigos Selva,

Maria del Carmen

Garrote, Gil

Garson, Mary

Gärtner, Peter

Gasbarri, Carla

Gaspar, Diaz

Gaspar, Krisztian

Gasparini, Pierliugi

Gasparri, Fabio

Gasparrini, Massimiliano
Gasser, Christoph

Gates, Will

Gautier, Arnaud

Gavara, Rafael

Gavicho Uarrota, Virgilio

Gavira, José A.

Gazerani, Parisa

Ge, Guanglu

Ge, Xiaodong

$\mathrm{Ge}$, Xiaowei

Geisler, Sarah

Geletii, Yurii V.

Gelmi, Maria Luisa

Gendaszewska-Darmach, Edyta

Genta-Jouve, Grégory

George, Riham F.

Georgiadi, Anastasia

Georgiev, Vasil

Geppetti, Pierangelo

Gerber, Doron

Gerke, Jörg

Germain, Marc

Germani, Raimondo

Geronikaki, Athina

Gerothanassis, Ioannis P.

Ghadwal, Rajendra S.

Ghanotakis, Demetrios F.

Ghavaminejad, Amin

Ghignone, Stefano

Giamperi, Laura

Giampieri, Francesca

Giampietro, Letizia

Gianferrara, Teresa

Giannoni, Patrizia

Giaouris, Efstathios

Gil, Ana

Gilabert-Oriol, Roger

Giles, Gregory

Gillinham, Dennis

Gillman, Jason

Gilmer, John F.

Gilson, Michael

Giménez, Raquel 
Gindensperger, Etienne

Giordano, Antonio

Giotta, Livia

Giovannoni, Maria P.

Giovarelli, Mirella

Girardon, Jean-Sébastien

Giraudeau, Patrick

Girbés, Tomás

Girlando, Alberto

Girousi, Stella

Gitzendanner, Matt

Giuntini, Francesca

Gnavi, Giorgio

Gohda, Eiichi

Goins, Beth

Goławska, Sylwia

Gold, Brian

Goldsmith, Chloe D.

Golmohamadi, Amir

Goltsov, Alexey

Gomes, Ana P.

Gómez, Ana María

Gómez, Rodolfo

Goncalves, Luis Moreira

Gonçalves, M. Sameiro T.

Gonçalves, Olivier

Gonda, Sándor

Gong, Junbo

Gong, Xingchu

Gonzales, Gerard Bryan

González, Concepción C.

González, Florenci V.

González Domínguez, Raúl

González-Andrade, Martín

González-Burgos, Elena Maria

González-Díaz, Humberto

González-Díaz, Oscar

González-Fernández, A.

González-Muñiz, Rosario

González-Sarrías, Antonio

Gorini Da Veiga, Ana

Gorman, Christopher B.

Gorman, Gregory S
Gorska, Magdalena

Górska, Sabina

Gossage, R. A.

Goto, Yuki

Gotor-Fernández, Vicente

Gouin, Sebastien

Goulas, Vlasios

Goulet, Charles

Gouws, Chrisna

Govind, Niranjan

Gozzo, Franco

Grace, Dr. Mary

Graf, Michael

Graikou, Dia

Gramza-Michałowska, Anna

Granado, M. Dolores

Grandfils, C.

Grant, George

Grassi, Alfonso

Gravel, Edmond

Gray, Christopher A.

Grée, René

Green, Don

Greenwood, David

Greffrath, Wolfgang

Gressent, Frédéric

Grider, Arthur

Grieco, Francesco

Grigalevicius, Saulius

Grilli, Selene

Grkovic, Tanja

Grooten, Johan

Gross, Aaron

Gross, Harald

Grossini, Elena

Grosso, Clara

Grosvenor, Andrew

Grøtli, Morten

Grovel, Olivier

Groysman, Stanislav

Gruber, Christian

Gruber, Jan

Grumet, Rebecca
Gruselle, Michel

Grynkiewicz, Grzegorz

Grynszpan, Flavio

Grzegorczyk-Karolak,

Izabela

$\mathrm{Gu}$, Jingkai

$\mathrm{Gu}$, Junfeng

Gu, Wenyi

$\mathrm{Gu}$, Zhen

Guan, Yongguang

Gudiminchi, Rama

Guerrero, Antonio

Guerrero, José

Guetschow, Michael

Gugliandolo, Concetta

Guiamet, Patricia

Guibal, Eric

Guidotti, Matteo

Guilhon, Giselle Maria S. P.

Guillou, Hervé

Guiné, Raquel P. F.

Gulati, Pankaj

Gulati, Vandana

Gunia-Krzyżak, Agnieszka

Gunness, Purnima

Guo, Fang

Guo, Fei

Guo, Fenghai

Guo, Hongchao

Guo, Huimin

Guo, Jia-Hsin

Guo, Pu

Guo, Yuan Qiang

Guo, Zheng

Gupta, Ankit

Gurevich, Eugenia V.

Gurramkonda, Chandrasekhar

Gustavsson, Thomas

Gutiérrez Martín, Santiago

Gutiérrez-Juárez, Roger

Gutiérrez-Uribe, Janet

Gutmann, Torsten

Guzik, Urszula 


\begin{tabular}{|c|}
\hline Guzmán, Fanny \\
\hline Guzzo, Flavia \\
\hline Gynther, Mikko \\
\hline Habata, Yoichi \\
\hline Habaue, Shigeki \\
\hline Habiba, Khaled \\
\hline Hachiya, Akira \\
\hline Hacker, Michael C. \\
\hline Hadjikakou, Sotiris K. \\
\hline Hadjipavlou-Litina, Dimitr \\
\hline Hagenlocher, Yvonne \\
\hline Hahn, Ekkehardt \\
\hline Haider, Norbert \\
\hline Halabalaki, Maria \\
\hline Haleagrahara, Nagaraja \\
\hline Hallett-Tapley, Geniece \\
\hline Hallsworth, J. E. \\
\hline Hamad-Schifferli, Kimberl \\
\hline Hamanishi, Junzo \\
\hline Hamberger, Bjoern \\
\hline Hamon, Jean-René \\
\hline Han, Jaehong \\
\hline Han, Jianlin \\
\hline Han, Quan-Bin \\
\hline Han, Sang-bae \\
\hline Han, Sang-Mi \\
\hline Han, Se Jong \\
\hline Han, Xiaoyuan \\
\hline Han, Zhengxu S. \\
\hline Hanaor, Dorian \\
\hline Hancock, Robert D. \\
\hline Handy, Scott \\
\hline Hanhineva, Kati \\
\hline Hano, Christophe \\
\hline Hanschen, Franziska S. \\
\hline Hansen, Espen \\
\hline Hansen, Niels \\
\hline Hanson, James R. \\
\hline Hanson, Robert N. \\
\hline Hanusa, Timothy \\
\hline Hao, Hongdong \\
\hline Hao, Xiaojuan \\
\hline Harada, Nobuyuki \\
\hline
\end{tabular}

Harbourne, Niamh

Hardeland, Rudiger

Hare, Joshua M.

Harenberg, J.

Harpke, Dörte

Harris, Philip

Harrison, Anna L.

Harrowfield, Jack

Harwood, Tim

Hasegawa, Morifumi

Hasegawa, Urara

Hashimoto, Makoto

Hashimoto, Naoto

Hashmi, A. Stephen K.

Hassan, Hassan M. A.

Hassan, Mohamed

Hassan, Yousef I.

Hatae, Noriyuki

Hatano, Tsutomu

Hattori, Haroldo T.

Haug, Bengt Erik

Hautbergue, Guillaume

Hay, Michael P.

Hayashi, Takashi

Hayes, Douglas G.

Haystead, Timothy

$\mathrm{He}$, Bin

He, Chengwei

$\mathrm{He}, \mathrm{Gu}$

He, Jun

Hearn, Michael J.

Hedtrich, Sarah

Heery, David M.

Hehlgans, Stephanie

Heidary, Damoon Sohrabi Baba

Heinrich, Timo

Heiskanen, Juha

Heitz, Valérie

Helena, Sovova

Helesbeux, Jean-Jacques

Helm, Richard

Hemming, Karl

Henderson, Bill
Henderson, Luke C.

Hendrich, Suzanne

Hennig, Andreas

Hensela, Andreas

Herdewijn, Piet

Hernández, Francisca

Hernández, Pablo E.

Hernández-Ledesma, Blanca

Herraiz, T.

Herrera, Raquel P.

Herrero, Laura

Herrmann, Fabian

Herrmann, Martin

Hervés-Beloso, Pablo

Hettiarachchi, D. S.

Hewitson, Peter

Hickman, James J.

Hidalgo, Dolores

Hidalgo Herrador,

José Miguel

Hidari, Kazuya

Hideg, Kálmán

Hiejima, Yusuke

Higuchi, Akon

Hingorani, Dina

Hintermann, Lukas

Hirakawa, Hideki

Hirakawa, Kazutaka

Hirano, Tomoya

Hirao, Ichiro

Hirasawa, Noriyasu

Hirayama, Fumitoshi

Hirche, Christoph

Hirota, Takashi

Hiroyuki, Tanaka

Hirsch, E.

Ho, Chi-Tang

Ho, Su-Chen

Hodge, David R.

Hofer, Tim

Hoff, Bård Helge

Hoffman, Angela M.

Hofmann, Bettina 


\begin{tabular}{|c|}
\hline Hofmann, Johann \\
\hline Hofmann, Ute \\
\hline Hohenegger, Martin \\
\hline Hojjat-Farsangi, Mohammad \\
\hline Holder, Alvin \\
\hline Holley, Richard A. \\
\hline Holm, Rene \\
\hline Holst, Otto \\
\hline Holt, Carl \\
\hline Holzmann, Nicole \\
\hline Hong, Guosong \\
\hline Hong, Yonggeun \\
\hline Hong, Yuning \\
\hline Hopkins, Samantha \\
\hline Höppener, Stephanie \\
\hline Horbowicz, Marcin \\
\hline Hori, Yuichiro \\
\hline Horowitz, John D. \\
\hline Horrigan, Louise A. \\
\hline Horvath, David P. \\
\hline Hosoya, Takamitsu \\
\hline Hossain, Mohammad Billal \\
\hline Hou, Aiqin \\
\hline Hou, Anfu \\
\hline Hou, Duen-ren \\
\hline Hou, Harvey \\
\hline Hou, Zhengqing \\
\hline Houbracken, Isabelle \\
\hline Houen, Gunnar \\
\hline Houston, Douglas \\
\hline Hsia, Shih-Min \\
\hline Hsiao, George \\
\hline Hsieh, Changwei \\
\hline Hsieh, Jen-chien \\
\hline Hsieh, Jung-Feng \\
\hline Hsieh, Pei-Wen \\
\hline Hsin, Kun-Yi \\
\hline Hsu, Shan-hui \\
\hline $\mathrm{Hu}$, Anming \\
\hline $\mathrm{Hu}$, Guochang \\
\hline $\mathrm{Hu}$, Jiang \\
\hline $\mathrm{Hu}$, MingKuan \\
\hline $\mathrm{Hu}$, Ping \\
\hline
\end{tabular}

$\mathrm{Hu}$, Teh-min

$\mathrm{Hu}$, Xinzhong

Hu, Yinling

Huang, A-mei

Huang, Chi-Chang

Huang, Chih-Ching

Huang, Chun-Yung

Huang, Feihe

Huang, Hsi-Ya

Huang, Hsu-Shan

Huang, Hui-Chi

Huang, Hui-Pei

Huang, Jianguo

Huang, Jiann-Jyh

Huang, Liang-Feng

Huang, Lin-Fang

Huang, Ling

Huang, Renhua

Huang, Ren-Yeong

Huang, Sheng-You

Huang, Tzou-Chi

Huang, Wei

Huang, Wei-Jan

Huang, Wen-Chung

Huang, Wen-Hsin

Huang, Wenlin

Huang, Ya-ling

Huang, Youju

Huang, Zhi

Hubicka, Urszula

Hübner, Florian

Huc, Ivan

Huczyński, Adam

Huefner, Antje

Hueso, Jose L.

Huh, Yong-Min

Hui, Kam M.

Hullar, Meredith

Hulme, Alison

Hulme, Christopher

Hung, Wei Hsuan

Hunt, Piper Reid

Hunter, Luke
Hunter, Roger

Huo, Fang Jun

Huq, Laila

Hussein, Ahmed A.

Hutchens, Michael P.

Hutton, Craig

Hwang, Bang Yeon

Hwang, In Koo

Hwang, Kyung-Ran

Hwang, Thomas I-Sheng

Hwu, Jih-Ru

Hymel, David

Hyun, Myung Ho

Iacomini, Marcello

Iafisco, Michele

Iannazzo, Daniela

Iannuccelli, Valentina

Iatrou, Hermis

Ibarra, David

Ibrahem, Ismail

Ientile, Riccardo

Igarashi, Kiharu

Iglesias, Luis E.

Iglesias, Maria Del Rosario

Ikai, Tomoyuki

Ikegaya, Naoki

Ikushiro, Shin-ichi

Iliopoulos, Konstantinos

Ilisz, István

Ilton, Eugene S.

Imperatore, Concetta

Inagaki, Hidetoshi

Inami, Keiko

Iniesta, Jesus

Inman, Sharon

Inokuchi, Tsutomu

Inoue, Masayuki

Inoue, Shigeyoshi

Inserte, Javier

Ioannou, Efstathia

Iohara, Daisuke

Iorizzi, Maria

Irakli, Maria 


\begin{tabular}{|c|c|c|}
\hline Isaza Martínez, José Hipólito & Janusz, Grzegorz & Jin, Tienan \\
\hline Isemura, Mamoru & Jaque, Pablo & Jing, Jing \\
\hline Ishida, Shintaro & Jara-Palacios, M. José & Jiráček, Jiří \\
\hline Ishikura, Minoru & Jardine, Anwar & Johansen, Steinar D. \\
\hline Ishola, Mofoluwake M. & Jarstfer, Michael Bruce & Johansson, Mikael P. \\
\hline Islam, Md Soriful & Jasinski, Marcin & Johler, Sophia \\
\hline Ismaili, Lhassane & Jasiński, Radomir & John, Varghese \\
\hline Isman, Murray & Jastrzebska, Beata & Jolkkonen, Jukka \\
\hline Isman, Murray B. & Jászay, Zsuzsa & Jones, Jessica L. \\
\hline Isshiki, Yoshiaki & Javadi, Ali & Jones, Simon \\
\hline Ita, Kevin & Jayant, Rahul Dev & Jones, William \\
\hline Ito, Satoshi & Jean, Ludovic & Jonnalagadda, \\
\hline Ito, Shingo & Jee, Jun-Goo & Sreekantha Babu \\
\hline Ito, Takeru & Jelen, Henryk & Jonnalagadda, Subash C. \\
\hline Ito, Takuya & Jenett-Siems, Kristina & Jordao, Antonio M. \\
\hline Iwaizumi, Masakazu G & Jenssen, Håvard & Jørgensen, Kåre \\
\hline Iwamori, Masao & Jeon, Songhee & Joshi, Bharat \\
\hline Iwaoka, Michio & Jeong, Jin Boo & Joule, John \\
\hline Iwasaka, Masakazu & Jeong, Lak Shin & Juan, M. Emília \\
\hline Iwasaki, Hironori & Jeong, Rae-Dong & Juang, Tzong-Yuan \\
\hline Iwasaki, Takashi & Jeong, Seon-Yong & Juarranz, Ángeles \\
\hline Izawa, Hironori & Jeong, Yoonhwa & Jugé, Sylvain \\
\hline Jaakola, Laura & Jeons, Sangmin & Jullian, Carolina \\
\hline Jablonski, Dr. Monica M. & Jerković, Igor & Jung, Byung Hwa \\
\hline Jablonski, Miroslaw & Jer-Yiing, Houng & Jung, Ho-Sup \\
\hline Jacob, Claus & Jeschke, Gunnar & Jung, Sang Hun \\
\hline Jacobsen, Elisabeth Egholm & Jesionowski, Teofil & Jung, Seunho \\
\hline Jaffrezic-Renault, Nicole & Jeszka-Skowron, Magdalena & Jung, Young-Suk \\
\hline Jäger, Martin & Jetten, Anton & Junier, Pilar \\
\hline Jagerovic, Nadine & Jha, Mukund & Junior, Walter A. Roman \\
\hline Jagiello, Karolina & $\mathrm{Ji}$, Bin & Jurczak, Janusz \\
\hline Jain, Rachit & Ji, Debin & Jurikova, Tunde \\
\hline Jaki, Birgit & Jia, Yi-Xia & Juszczak, Lesław \\
\hline Jakočiūnas, Tadas & Jiang, Changyun & Jux, Norbert \\
\hline James, Samet & Jiang, Chaoyang & Jyh-fei, Liao \\
\hline Jan, Tong Rong & Jiang, Deli & Kachlicki, Piotr \\
\hline Jandrić, Zora & Jiang, K. E. & Kaczor, Agnieszka \\
\hline Jang, Dae Sik & Jiang, Shu-Ye & Kadam, Shekhar \\
\hline Jang, Ik-Soon & Jiang, Zhiyong & Kadokawa, Jun-ichi \\
\hline Jang, Jaehyuk & Jiménez Blanco, José L. & Kador, Lothar \\
\hline Jankowska-Anyszka, Marzena & Jiménez-Araujo, Ana & Kadota, Isao \\
\hline Jansa, Josef & Jimeno, Ciril & Kahlert, Stefan \\
\hline Jansen, Rolf & Jin, Jiefu & Kaipa, Ushasree \\
\hline
\end{tabular}


Kakkar, Ashok K.

Kalinowska-Lis, Urszula

Kalinowski, Danuta S.

Kalska-Szostko, B.

Kamdem, D. Pascal

Kamiloglu, Senem

Kanai, Yoshikatsu

Kanakkanthara, Arun

Kanchiswamy,

Chidananda Nagamangala

Kanda, Yasuharu

Kang, ChanKyu

Kang, Chulhun

Kang, Dong-Ku

Kang, Jae Seung

Kang, Jihee Lee

Kang, Jong Seong

Kang, Jonghoon

Kang, Jun Yong

Kang, Sinyoung

Kang, SO

Kang, Sun Chul

Kanie, Osamu

Kankanala, Jayakanth

Kano, Mitsuyoshi

Kao, Chai-Lin

Kapalavavi, Brahmam

Kappel, Christian

Kapusta-Duch, Joanna

Karafiloglou, Padeleimon

Karalekas, Dimitris

Karamanis, Panaghiotis

Karamaouna, Filitsa

Karczmarczyk, Aleksandra

Kärkönen, Anna

Karlic, Heidrun

Karmakar, Partha

Karnaouri, Anthi

Karoglan, Marko

Karpoormath, Rajshekhar

Karsili, Tolga

Kaschula, Catherine H.

Kashfi, Khosrow
Kashiwada, Yoshiki

Kashman, Yoel

Katagiri, Hiroshi

Kataoka, H.

Kataoka, Hiromi

Kataoka, Takao

Katayama, Shigeru

Kath-Schorr, Stephanie

Kathuria, Himanshu

Katiyar, Santosh K.

Kato, Atsushi

Kato, Koichi

Kato, Masaya

Katoh, Tadashi

Katsila, Theodora

Katsuzaki, Hirotaka

Katuchova, Jana

Kaufman, Teodoro S.

Kaufmann, Dorothea

Kauppinen, Anu

Kavallieratos, Nickolas G.

Kawabata, Kyuichi

Kawahara, Teppei

Kawai, Yoshichika

Kawakami, Susumu

Kawakita, Hidetaka

Kawamura, Akira

Kawamura, Fumio

Kawao, Naoyuki

Kawashima, Hideki

Kawashima, Takayuki

Kay, Colin D.

Kay, Graeme

Kayaki, Yoshihito

Kelesidis, Theodoros

Kelly, Ryan T.

Kerr, William L.

Keserü, György M.

Keski-Rahkonen, Pekka

Keski-Saari, Sarita

Kessel, David

Kessel, Line

Keul, Helmut
Keum, Gyochang

Keum, Young Soo

Kevil, Christopher G

Keyzers, Rob

Khalid, Sami A.

Khan, Imran

Khan, Mohsin

Khan, Muhammad Iqbal

Khenoussi, Nabyl

Khulbe, K. C.

Khun, Nay Win

Kiba, Akinori

Kibayashi, Kazuhiko

Kiec-Kononowicz,

Katarzyna

Kiefer, Christiane

Kietzmann, Thomas

Kievit, Forrest M.

Kikuchi, Haruhisa

Kim, Byung-Soo

Kim, Chul Young

Kim, Chun Ho

Kim, Chu-Young

Kim, Dong-Hyun

Kim, Donguk

Kim, Gheun-Ho

Kim, Han-do

Kim, Hyemee

Kim, Hyung Kwoun

Kim, Hyung Sik

Kim, Hyun-Joong

Kim, Hyunwoo

Kim, Jae Young

Kim, Jaheon

Kim, Ji Yeon

Kim, Jin-Kyung

Kim, Jong-Ho

Kim, Joong Sun

Kim, Joungmok

Kim, Jung-Ae

Kim, Junghyun

Kim, Kikang

Kim, Kil-Soo 


\begin{tabular}{|c|}
\hline Kim, Kyu-Bong \\
\hline Kim, Kyung-su \\
\hline Kim, Myeong Ok \\
\hline Kim, Sejoong \\
\hline Kim, Seok-Jhin \\
\hline Kim, Seung-Whan \\
\hline Kim, Sung Yeon \\
\hline Kim, Sung-Hoon \\
\hline Kim, Sunjoo \\
\hline Kim, Tae Kon \\
\hline Kim, Tae-Jeong \\
\hline Kim, Yang \\
\hline Kim, Yeon-Ju \\
\hline Kim, Young Sang \\
\hline Kim, Young-jae \\
\hline Kim, Young-Kyoon \\
\hline Kim, Youngmee \\
\hline Kim, Young-Woo \\
\hline Kim, Yujin \\
\hline Kim, Yun-bae \\
\hline Kimura, Ken-ichi \\
\hline Kinaciyan, Tamar \\
\hline Kinder, David \\
\hline King, Michael \\
\hline Kinoshita, Takayoshi \\
\hline Kinthada, Ramakumar \\
\hline Kiparissides, Costas \\
\hline Kirby, Christopher W. \\
\hline Kirsch, \\
\hline Professeur Emérite Gilbert \\
\hline Kirton, Stewart B. \\
\hline Kiss, Robert \\
\hline Kitagawa, Wataru \\
\hline Kitagishi, Hiroaki \\
\hline Kitamura, Masanori \\
\hline Kitazawa, Takafumi \\
\hline Kiyama, Ryoiti \\
\hline Klajnert, B. \\
\hline Klaus, Susanne \\
\hline Klausen, R. S. \\
\hline Klaveness, Jo \\
\hline Klein, Christian \\
\hline Klempt, Martin \\
\hline
\end{tabular}

Klettner, Alexa

Kluepfel, Daniel

Kluger, Ronald

Klüß, Jeannette

Knölker, Hans-Joachim

Knoshaug, Eric

Knupp, Gerd

Ko, Kam Ming

Ko, Shun-Yao

Ko, Young Tag

Kobayashi, Kazuhiro

Kobayashi, Makoto

Kobayashi, Nobuyuki

Kobets, Tetyana

Kobuchi, Shinji

Kochi, Takuya

Kocsis, Bela

Kogure, Noriyuki

Koh, Hwee Ling

Kohler, Bern

Koivisto, Ari

Kojima-Yuasa, Akiko

Kojo, Shosuke

Kok, Victor C.

Kolb, Peter

Kolbert, Zsuzsanna

Kolhar, Poornima

Kollár, László

Kolodkin-Gal, Ilana

Komeiji, Yuto

Komori, Kikuo

Konaté, Kiessoun

Kong, In-soo

Kong, Siu-Kai

König, Burkhard

Konkolewicz, Dominik

Kooyman, P. J.

Kopel, Pavel

Koppel, Kadri

Kortz, Ulrich

Koschella, Andreas

Kosmrlj, Janez

Kosova, Klara
Kostakis, Ioannis

Kostomitsopoulos,

Nikolaos G.

Kotloski, Robert

Kotsikorou, Evangelia

Koufaki, Maria

Koul, Opender

Koulman, Albert

Koundouras, Stefanos

Kounis, Nicholas G.

Kourkoutas, Yiannis

Koutentis, Panayiotis

Kouvelis, Vassili N.

Kovacs, Lajos

Kovács, Ákos T.

Kowalewski, Markus

Kowalski, Konrad

Kowalski, Radosław

Koziel, Jacek

Kozliak, Evguenii I.

Kozłowski, Henryk

Kozono, David

Kraaijeveld, Ken

Krajewska, Barbara

Krajka-Kuźniak, Violetta

Královec, Karel

Krämer, Stefanie

Kraus, Birgit

Krchňák, Viktor

Kregel, Steven

Kreis, Wolfgang

Kren, Vladimir

Kresh, J. Yasha

Kriegsheim, Alex Von

Kries, Hajo

Kuan, Yu-Hsiang

Kubicek, Christian P.

Kubíček, Vojtěch

Kubicova, Lenka

Kubina, Robert

Kubota, Naoto

Kuca, Kamil

Kucerik, Jiri 
Kucuk, Israfil

Kujawski, Wojciech

Kumar, Ashutosh

Kumar, Gaurav

Kumar, Naresh

Kumar, Ujendra

Kuno, Toshiya

Kunz, Wolfram

Kunze, Gotthard

Kuo, Ping-Chung

Kurakevych, Oleksandr O.

Kurdowska, Anna K.

Kurokawa, Ichiro

Kus, Piotr Marek

Kuwahara, Masayasu

Kuzma, Lukasz

Kuz'min, Victor

Kvasnica, Miroslav

Kwak, Jong-Young

Kwan, Hiu-yee

Kwatra, Deep

Kwon, Il-Keun

Kwon, Oran

Kwon, Sung Won

Kwon, Young-Wan

Kwong, Fuk Yee

Kyasa, Shiva Kumar

Kyung, Kyu Hang

Kyushin, Soichiro

La Motta, Concettina

La Regina, Giuseppe

Laali, Kenneth

Laatsch, Hartmut

Lacaille-Dubois, Marie-Aleth

Laczka, Csilla

Ladero, Miguel

Ladero Galán, Miguel

Laemmerhofer, Michael

Lago, N. Faginas

Lahav, Ori

Lahooti, Hooshang

LaJeunesse, Dennis R.

Lam, Yulin
Lamprou, Dimitrios

Lamuela Raventos, Rosa

Lan, Chung-Yu

Lan, Tian

Landete, José Maria

Lane, Majella

Lang, Jian-Ping

Lange, Heiko

Langeveld, Hans

Laport, Marinella

Laquintana, Valentino

Larraneta Landa, Eneko

Latortue, Marie C.

Latosińska, Jolanta Natalia

Lattuada, Marco

Lau, Christopher S.

Lau-Cam, Cesar A.

Laurent, FERRIÉ

Lauro, M. R.

Lavandera, Ivan

Lavarda, Francisco Carlos

Lavrentovich, Oleg D.

Law, Betty Yuen-Kwan

Lawen, Alfons

Lazzarato, Loretta

Lazzaroni, Roberto

Le Grognec, Erwan

Le Hégarat, Ludovic

Le Pape, Patrice

Le Pogam, Pierre

Leach, Katie

Leadbeater, Nicholas

Lechtenberg, Matthias

Leclercq, Guy

Leclère, Philippe

Ledoigt, Gérard

Lee, Adam

Lee, Bun Yeoul

Lee, Chang Hoon

Lee, Chang-Hun

Lee, Che-Hsin

Lee, Chin-Fa

Lee, Ching-Kuo
Lee, Chong-Kil

Lee, Choon Young

Lee, Choong Hwan

Lee, Dong Gun

Lee, Dong-Chan

Lee, Dongwon

Lee, Eun-Hee

Lee, Guem-San

Lee, Hee Soon

Lee, Hon Man

Lee, Huei-Jane

Lee, Hwa Jin

Lee, Hye Suk

Lee, Jae Wook

Lee, Jae Yeol

Lee, Jaehwi

Lee, Jeongmi

Lee, Jin-Koo

Lee, Jiun-haw

Lee, Jiyoun

Lee, Jonghwi

Lee, Jongkook

Lee, Jong-Seok

Lee, Jun

Lee, Jun Sik

Lee, Junsoo

Lee, Kin Wah Terence

Lee, Kwang Ho

Lee, Lin-wen

Lee, Meng-Jen

Lee, Mi Kyeong

Lee, Min Won

Lee, Mina

Lee, Myung G.

Lee, Sang Kook

Lee, Sang Kyu

Lee, Sang-han

Lee, Seong-Wook

Lee, Seung Hee

Lee, Shyi-Long

Lee, So Yeong

Lee, Sunwoo

Lee, Tae Soo 


\begin{tabular}{|c|c|c|}
\hline Lee, Tai-Lin & Li, Chunshun & Liang, Xin-miao \\
\hline Lee, Tse-Min & Li, Chunxi & Liang, Yong \\
\hline Lee, $\mathrm{Tu}$ & Li, Dongmei & Liang, Yuerong \\
\hline Lee, Tzong-shyuan & Li, Dongye & Liao, Ai-Ho \\
\hline Lee, Wing-Hin & Li, Guijie & Liao, Daiqing \\
\hline Lee, Yong Rok & Li, HouJin & Liao, Hui-Fen \\
\hline Lee, Yueh-Lun & Li, Huabin & Liao, Peng \\
\hline Leeper, Finian J. & Li, Hui & Liaw, Chih-Chuang \\
\hline Legentil, Laurent & Li, Jie & Lidon, Fernando Cebola \\
\hline Lehnherr, Dan & $\mathrm{Li}, \mathrm{Li}$ & Lieberzeit, Peter \\
\hline Lei, Aiwen & Li, Mei-Ling & Liermann, Johannes C. \\
\hline Lei, Dangyuan & Li, Nianguang & Liew, Chongwee \\
\hline Lei, Weiwei & Li, Pengcheng & Lifshitz, Efrat \\
\hline Leiherer, Andreas & Li, Ping & Ligon, Lee A. \\
\hline Leisner, Jørgen J. & Li, Qingliang & Lill, Markus A. \\
\hline Lekka, Malgorzata & Li, Rui & Lim, Chee \\
\hline Lemus, Jesús & Li, Shao-Shun & Lim, Dong Wook \\
\hline Len, Christophe & Li, Shujun & Lim, Dong-Kwon \\
\hline Lenardão, Eder J. & Li, Siheng & Lim, Yoongho \\
\hline Lenoir, Irene & Li, Tiejun & Lima, Raquel T. \\
\hline Lentini, Giovanni & Li, Wei-lin & Lima-Filho, José V. M. \\
\hline Leondaritis, George & Li, Weirong & Lin, Chih-Li \\
\hline Leonetti, Francesco & Li, Wen-Wu & Lin, Dr. Henry C. \\
\hline Leong, David Tai & Li, Xian & Lin, Hai-shu \\
\hline Leoni, Alberto & Li, Xiang-Guo & Lin, Hui \\
\hline Leonidas, Demetres D. & Li, Xiaobo & Lin, Hung-Yin \\
\hline Lephart, Edwin D. & Li, Xiyou & Lin, Kuo-Shyan \\
\hline Leporatti, Stefano & Li, Yimeng & Lin, Liang-Tzung \\
\hline Lerm, Maria & Li, Yingxia & Lin, Li-Gen \\
\hline Lescrinier, Eveline & Li, Yinyin & Lin, Shangchao \\
\hline Lesyk, Roman & Li, Yongle & Lin, Shyh-Hsiang \\
\hline Leung, Bernard & Li, Yuliang & Linert, Wolfgang \\
\hline Leung, Chung-Hang & Li, Zhen & Ling, Chen \\
\hline Leung, Elaine Lai-Han & Li, Zhi-Minhg & Ling, Yong-Chien \\
\hline Leung, Ivanhoe & Li, Zhongjing & Link, Wolfgang \\
\hline Leung, Ken & Li, Xu-Wen & Linz, John E \\
\hline Levy, Arthur & Liagre, B. & Liou, Chian-Jiun \\
\hline Lewinska, Anna & Liakos, Ioannis L. & Liou, Ying-ming \\
\hline Lewis, Karen & Liang, Dong & Lisboa, Patrícia \\
\hline Lewkowicz, Elizabeth & Liang, Kang & Lisi, Luciana \\
\hline Lewkowski, Jaroslaw & Liang, Li & Litinas, Konstantinos E. \\
\hline Leyssens, Tom & Liang, Po-huang & Little, R. Dan \\
\hline Lhiaubet, Virginie Lyria & Liang, Shih-Shin & Liu, Baoming \\
\hline
\end{tabular}


Liu, Bifeng

Liu, Cheuk Lun

Liu, Chong

Liu, Chun-yan

Liu, Dajiang

Liu, Delong

Liu, Fei

Liu, Guijian

Liu, Hai-yang

Liu, Hong

Liu, Hongbing

Liu, Hui-ling

Liu, Jin

Liu, Jin-Gang

Liu, Jun-Jen

Liu, Lei

Liu, Liang

Liu, Liangliang

Liu, Peng

Liu, Renyu

Liu, Shih-Jung

Liu, Shing-Hwa

Liu, Shiuh-Tzung

Liu, Xiaojing

Liu, Xiaoxi

Liu, Xin

Liu, Yanfang

Liu, Ying-Ling

Liu, Yonghong

Liu, Yongqiang

Liu, Yuan-Shuai

Liu, Yue

Liu, Yujun

Liu, Zhi Long

Liu, Jialei

Lizcano De Vega, José Miguel

Llona-Minguez, Sabin

Lloyd-Williams, Paul

Lo, Kenneth Kam-Wing

Lo, Lee-Chiang Lo

Lo Scalzo, Roberto

Locatelli, Marcello

Loh, Xian Jun
Loizzo, Monica Rosa

Lok, Chun Nam

Lombó, Felipe

Longo, Pasquale

Longo, Vincenzo

Longstaffe, James G.

Loos, Katja

Lopez, Dolores E.

Lopez, Luis

Lopez, Victor

López, José Manuel

López, María L.

López-Abán, Julio

López-Bucio, J.

Lopez-Garzon, Rafael

Lopez-Lluch, Guillermo

Loppi, Stefano

Lord, Megan

Lorenzi, Vannina

Loris, Remy

Loschen, Christoph

Lou, Song

Loveridge, Joel

Lowary, Todd

Lu, Chung-kuang

Lu, FaChuang

$\mathrm{Lu}$, Jun

Lu, Jyh-Feng

Lu, Mei-Chin

Lu, Ming

Lu, Qi-long

$\mathrm{Lu}$, Shuo

Lu, Yongen

Lucas, David M.

Lucini, Luigi

Ludwiczuk, Agnieszka

Lugemwa, Fulgentius Nelson

Lui, Edmund M.K.

Luis, Santiago V.

Łukasik, Rafał

Lukyanov, Pavel A.

Luna, A.s.

Luna, Jonny E. Duque
Lundén, Karl

Lung, Ming-Yeou

Luo, Jian

Luo, Qi Cong

Luo, Shi-Zhong

Luo, Yinggang

Lupiáñez, José A.

Luque, F. Javier

Lustri, Wilton

Lyons, Russell

Ma, Choong Je

Ma, Guiping

Ma, Xiaoqian

Ma, Yong

Ma, Yueming

Maas, Michael

Maccallini, Cristina

Macchi, Beatrice

Macedo, Maria Lígia

Machetti, Fabrizio

Maciejewska, Dorota

Maciel, Patrícia

Mackerell, Alexander D.

Macknight, Richard C.

Mackowski, Sebastian

Maçôas, Ermelinda

Madar, Zecharia

Maddau, L.

Madesis, Panagiotis

Madsen, Andreas S.

Maeda, Hayato

Maeda, Katsuhiro

Maestro, Miguel

Mafra, Isabel

Magalhães, Júlia M. C. S.

Magata, Yasuhiro

Maggi, Filippo

Magnaldo, Thierry

Maguire, Anita

Maher, Pamela

Mahmudov, Kamran

Maicas, Sergi

Maier, Camelia 
Maier, Jeanette A.m.

Maier, Patrick

Maione, Francesco

Maisch, Tim

Majumdar, Susruta

Mak, King Lun Kingston

Mäkelä, Jyrki M.

Makihira, Seicho

Mäkinen, Sari

Makishima, Makoto

Makosza, Mieczyslaw

Malachová, Alexandra

Malaguarnera, Michele

Malawska, Barbara

Malda, Jos

Malde, Alpesh

Maldini, Mariateresa

Male, David K.

Malheiro, Ricardo

Malkoch, Michael

Mallipeddi, Prema Latha

Maloney, Mark

Malucelli, Giulio

Malumbres, Marcos

Mamane, Victor

Mambu, Lengo

Man, Dula

Manabe, Shino

Manca, Gabriele

Manca, Maria Letizia

Mancheño, José

Mancheño, Olga Garcia

Mancini, Ines

Manda, Vamshi K.

Mandal, Debashis

Mañes, Jordi

Mang, Thomas S.

Mangelinckx, Sven

Mannino, Saverio

Mano, Junichi

Mano, Yuji

Manos, Manolis

Manrique, Camila
Mansfield, Anna Katharine

Mantovani, Giuseppe

Manzo, Emiliano

Mao, Chuanbin

Mao, Zong-Wan

Marakos, Panagiotis

Marc, Daniel

Marcelli, Augusto

Marchand, Pascal

Marchiani, Sara

Marchi-Delapierre, Caroline

Marco-Contelles, José

Marcos, Mercedes

Marcotullio, Carla

Marczak , Jacek

Maresca, Marc

Marestin, Catherine

Márialigeti, Károly

Mariappan, Kadarkaraisamy

Marin, D.E.

Marin, Jose J. G.

Marini, Francesca

Marinkovic, Nebojsa

Marinozzi, Maura

Markopoulos, John

Markopoulou, Olga

Markossian, Sarine

Markovitsi, Dimitra

Maroja, Luana S.

Marques, Claudia

Marquetand, Philipp

Marra, Alberto

Marrocchi, Assunta

Marschner, Christoph

Marsh, Lorraine

Marsura, Alain

Marti, Guillaume

Martin, Jessica

Martin, Julia

Martin, Mathew

Martín, Juan F.

Martín-Banderas, Lucía

Martinelli, Adriano
Martínez, Alberto

Martínez-Palou, Rafael

Martin-Ramos, Pablo

Martins, Sonia

Martin-Sampedro, Raquel

Martra, Gianmario

Maruyama, Kei

Marverti, Gaetano

Marzaro, Giovanni

Marzocco, Stefania

Masaoka, Shigeyuki

Mase, Nobuyuki

Masek, Anna

Maserti, Bianca Elena

Massa, Antonio

Massaro, Marika

Masterson, Douglas

Mastrangelo, Anna M.

Masu, Hyuma

Masurier, Nicolas

Mata, I.

Matar, Samir F.

Mateos-Timoneda, Miguel A.

Materić, Dušan

Materska, Małgorzata

Mathesius, Ulrike

Mathieu, Didier

Matiadis, Dimitris

Matin, Abdul

Matosiuk, Dariusz

Matsubara, Keiichi

Matsuda, Hisashi

Matsugo, Seiichi

Matsui, Kenji

Matsui, Masaki

Matsui, Nobuaki

Matsui, Toshiro

Matsui-Yuasa, Isao

Matsumoto, Kazuya

Matsumoto, Yasuhiko

Matsumura, Kiyoshi

Matsumura, Shuichi

Matsuno, Koji 


\begin{tabular}{|c|c|c|}
\hline Matsuoka, Shin-ichi & Melone, Lucio & Miki, Yasuhiro \\
\hline Matsuzaki, Yasushi & Melzig, Matthias & Miklossy, Gabriella \\
\hline Mattivi, Fulvio & Memarzadeh, Kaveh & Mikołajczyk-Bator, \\
\hline Mattoo, Autar & Mena-Rejon, Gonzalo J. & Katarzyna \\
\hline Maurel, Marie-christine & Menendez Ramos, José Carlos & Mikulski, Damian \\
\hline Mavila, Sudheendran & Menet, Marie Claude & Milanesio, Marco \\
\hline Mavromoustakos, Thomas & Menezes, José & Milcarek, Christine \\
\hline Mayence, Annie & Meng, Huan & Milella, Luigi \\
\hline Mayhan, William G. & Menkhorst, Ellen & Milelli, Andrea \\
\hline Mazerska, Zofia & Menna, Marialuisa & Miller, John \\
\hline Mazzeo, Marco & Menut, Paul & Miller, Marshall G. \\
\hline Mazzola, Eugene & Mercier-Bonin, Muriel & Millet, Oscar \\
\hline Mazzone, Gloria & Mercolini, Laura & Millo, Enrico \\
\hline Mazzoni, Luca & Merfort, Irmgard & Mimaki, Yoshihiro \\
\hline Mbosso, & Merino, Pedro & Mimica-Dukic, Neda \\
\hline Emmanuel Jean Teinkela & Merlini, Lucio & Minbiole, Kevin P. C. \\
\hline Mcardle, Patrick & Merlino, Antonello & Mindt, Thomas L. \\
\hline McCallum, Jason L. & Merlo, Aloir Antonio & Minguillón, Cristina \\
\hline McCann, Mark J. & Mero, Anna & Minutolo, Filippo \\
\hline Mccarley, Robin L. & Mesías García, Marta & Miranda, Cristobal \\
\hline McCormick, Alon V. & Messeguer, Angel & Miranda, Katrina \\
\hline McFeeters, Robert L. & Messina, Federica & Miron, Richard J. \\
\hline Mcilroy, David N. & Mestres, Adrià Gil & Mirzayans, Razmik \\
\hline McIntosh, Marla S. & Métro, Thomas-Xavier & Misaki, Tomonori \\
\hline McLemore, Gabrielle & Metzinger, Laurent & Mishra, Vandana \\
\hline McNamara, Bruce & Meulia, Tea & Mitakou, Sofia \\
\hline McPhail, Kerry & Mewis, Inga & Mitchell, Jane \\
\hline McRae, Jacqui M. & Meyer, Hans-Peter & Miura, Tsuyoshi \\
\hline Meagher, Laurence & Meyers, Marvin J. & Miyabe, Hideto \\
\hline Meagher, Robert & Mező, Gábor & Miyamoto, Koji \\
\hline Mebs, Dietrich & Mezzenga, Raffaele & Miyamoto, Manabu \\
\hline Medana, Claudio & Mhd Jalil, Abbe Maleyki & Miyazaki, Toshiki \\
\hline Medeiros, Rodrigo & Michalcova, Lenka & Miyazato, Hironari \\
\hline Medici, Serenella & Michiels, Carine & Mizuno, Cassia S \\
\hline Medvidović-Kosanović, & Micura, Ronald & Mizuno, Masashi \\
\hline Martina & Midgley, Adam & Mizushige, Takafumi \\
\hline Mehbub, Mohammad Ferdous & Miecznikowski, K. & Mizutani, Osamu \\
\hline Mehta, Akul Y. & Miele, Adriana E. & Mladěnka, Přemysl \\
\hline Mei, Xuefeng & Migone, Aldo & Mlcek, Jiri \\
\hline Meille, Valérie & Miguel, Graça & Młochowski, Jacek \\
\hline Meinke, Martina & Míguez, Hernán & Mlsna, Todd \\
\hline Melino, Sonia & Mija, Alice & Moal, Iain \\
\hline Melo, André & Mikata, Yuji & Mohamed, Ramdani \\
\hline
\end{tabular}




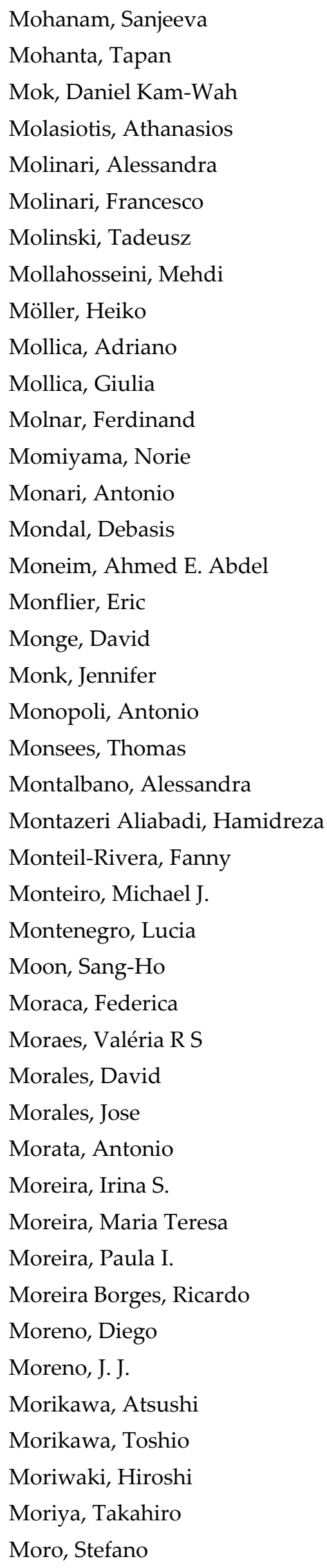

Morris, Gordon A.

Morris, James C.

Morse, Stephen

Moschos, Sterghios A.

Mostofian, Barmak

Mota, Manuel

Motoyama, Keiichi

Motte, Laurence

Motz, Günter

Moudgil, Kamal D.

Mourtzinos, Ioannis

Moussa, Fathi

Moyano, Albert

Moyano, Lourdes

Mózsik, Gyula

Mphahlele, Malose Jack

Muccioli, Giulio G.

Mueller, Geoffrey A.

Muhle-Goll, Claudia

Mühlenhoff, Ulrich

Mukherjee, Jogeshwar

Mukherjee, Subhendu

Mukudai, Yoshiki

Muller, M.

Muller, Ulrich

Müller, Christoph

Müller, Karsten

Müller, Werner

Mulloy, Barbara

Mulo, Paula

Mun, Jiyoung

Munoz, Macarena

Murai, Junko

Murai, Masahito

Murali, D.

Muranaka, Atsuya

Murkovic, Michael

Murray, Deirdre M.

Murthi, Padma

Murugaiyan, Jayaseelan

Murugan, N. Arul

Musch, Mark

Musiol, Robert
Musso, Loana

Mustafa, Arwa

Mutus, Bulent

Mykhaylyk, Olga

Myllykallio, Hannu

$\mathrm{Na}$, Dong Hee

Nachtigallová, Dana

Nagai, Takayuki

Nagai, Yoshinori

Nagaoka, Satoshi

Nagasawa, Kazuo

Nagata, Koji

Nagata, Toshi

Nagatomo, Shigenori

Nagulapalli Venkata,

Kalyan

Nagy, Peter

Naha, Pratap C.

Nair, Vimal

Nakagawa-Goto, Kyoko

Nakamura, Itaru

Nakamura, Kozo

Nakamura, Noriko

Nakanishi, Ikuo

Nakano, Koji

Nakashima, Souichi

Nakatani, Yoshihiko

Nalda-Molina, Ricardo

Nam, Ki Tae

Namiesnik, Jacek

Nandha Premnath,

Padmavathy

Nanjo, Fumio

Napoleão, Thiago Henrique

Nardi, Monica

Nasri, Rim

Nassir, Fatiha

Natalello, Antonino

Naumova, Anna V.

Navarra, Michele

Navarro, Daniela M. A. F.

Nawrocka, Agnieszka

Nazaré, Marc 
Nazarewicz, Rafal

Nazaruk, Jolanta

Nazzaro, Filomena

Nebigil, Canan G.

Nedachi, Taku

Neely, Brian J.

Negri, Fabrizia

Neilson, Andrew

Nekkanti, Vijaykumar

Nekongo, Emmanuel E.

Nemoto, Kiyomitsu

Nemykin, Victor

Nenadis, Nikolaos

Nerín, Cristina

Nesmelova, Irina V.

Nesterov, Dmytro

Neumann, Terrence

Neunert, Grazyna

Neureiter, Daniel

Nevárez-Moorillón,

Guadalupe Virginia

Neves, M. Graça P.M.S

Newman, David

Newmaster, Prof. Steven G.

$\mathrm{Ng}$, Li Fang

$\mathrm{Ng}$, Tzi Bun

Ngo, Huynh Thien

Nguyen, Minh Tho

Nguyen, Tuyen

Nicklaus, Marc C.

Nicoletti, Rosario

Nielsen, Henrik

Nielsen, Line Hagner

Nielsen, Mogens Brøndsted

Nielsen, Peter E.

Niemantsverdriet, J. W.

Nieuwenhuys, Bernard

Nihei, Ken-ichi

Niikura, Kenichi

Nikas, Spyros P.

Nikitenko, Sergey I.

Nikolakakis, Ioannis

Nippe, Michael
Nishikata, Takashi

Nishiwaki, Hisashi

Nishizawa, Seiichi

Nitulescu, George Mihai

Niu, Xiao D.

Niwa, Toshio

Niwano, Yoshimi

No, Joo Hwan

Nobili, Stefania

Noël, Timothy

Noge, Koji

Noh, Jeong Sook

Nolte-'t Hoen, Esther N. M.

Nomikos, Tzortzis

Nomura, Yoshihiro

Nonami, Atsushi

Nonappa, Nonappa

Normand, Philippe

Norvez, Sophie

Notarangelo, Francesca

Notni, Johannes

Novella, Susana

Novello, Vittorino

Novy, Pavel

Nowak, Renata

Nowak, Sascha

Nowak-Sliwinska, Patrycja

Nowicka, Anna Maria

Nowicki, Janusz

Ntalli, Nikoletta G.

Ntie-Kang, Fidele

Nuhn, Lutz

Nycz, Jacek E

Nyokong, Tebello

Nyulász, László

O'Connell, Mary A.

Obenland, David

Oberholster, Anita

Obied, Hassan

Oda, Akifumi

Oda, Tatsuya

Odell, Luke

O'Doherty, George
Oestreich, Martin

Oga, Enoche F.

Ogawa, Akiya

Ogawa, Noriko

Ogawa, Shojiro

Ogihara, Takuo

Ogino, Shuji

Oh, Jae-Min

Oh, Jung Hwan

Oh, Kyung Wha

Oh, Seikwan

Oh, Sei-Ryang

Oh, Won Keun

Ohisa, Satoru

Ohmura, Koichiro

Ohnishi, Masatoshi

Ohshima, Tatsuya

Ohshima, Tomoko

Ohta, Masaya

Ohta, Shinji

Ohta, Yoshiji

Oikawa, Akira

Oikawa, Hideaki

Ojika, Makoto

Okada, Taketo

Okafor, Florence

Okamura, Nobuyuki

Okazawa, Atsushi

Okino, Tatsufumi

Okumura, Tadayoshi

Oledzka, Ewa

Olenyuk, Bogdan Z.

Oliva, Romina

Oliveira, M. Beatriz P. P.

Oliveira, P.A.

Oliveira Rocha,

Hugo Alexandre

Oliverio, Manuela

Olivieri, Giuseppe

Olson, Kenneth

Olson, Mark A.

Omae, Iwao

Omar, Syed Haris 


\begin{tabular}{|c|}
\hline Omonov, Tolibjon \\
\hline Omoruyi, Felix \\
\hline Omosa, Leonidah K. \\
\hline Onduka, Toshimitsu \\
\hline Onnis, Valentina \\
\hline Ooyama, Yousuke \\
\hline Opatz, Till \\
\hline Operamolla, Alessandra \\
\hline Orecchio, Santino \\
\hline Ortega, José \\
\hline Ortega, Maria Teresa \\
\hline Ortinski, Pavel I. \\
\hline Ortiz, Fernando López \\
\hline Ortiz Sainz de Aja, Alfredo \\
\hline Ortiz-Mellet, Carmen \\
\hline Ortiz-Urquiza, Almudena \\
\hline Osborn, Helen \\
\hline Osbourn, Anne \\
\hline Ospina Millan, Claudia \\
\hline Ostareck, Dirk H. \\
\hline Othman, Zeid A. Al \\
\hline Ottonelli, Massimo \\
\hline Oyewumi, Moses \\
\hline Ozawa, Fumiyuki \\
\hline Ozeki, Yasuhiro \\
\hline Pacifico, Severina \\
\hline Padmalayam, Indira \\
\hline Padovan, Amanda \\
\hline Paduch, Roman \\
\hline Paeng, Ki-Jung \\
\hline Pagliaro, Pasquale \\
\hline Paik, Hyun-dong \\
\hline Pak, Sok Cheon \\
\hline Pakulski, Zbigniew \\
\hline Pal, Rituraj \\
\hline Palacios, Sara \\
\hline Pałasz, Aleksandra \\
\hline Palazon, Javier \\
\hline Palazzo, Enza \\
\hline Palenzuela López, \\
\hline José Antonio \\
\hline Paller, Channing \\
\hline Palm, Gottfried J. \\
\hline
\end{tabular}

Palomar, Manuel

Palumbo, Anna

Pan, Tai-long

Pan, Tzu-Ming

Pan, Wei-Ping

Panacek, Aleš

Panaro, Maria Antonietta

Panek, Jarosław

Paneth, Agata

Panfoli, Isabella

Pantazis, Dimitrios A.

Panuwet, Parinya

Panzella, Lucia

Paola, Luisa Di

Paola, Stiuso

Paoli, Paola

Paoli, Paolo

Paolone, Annalisa

Papadimitriou, Vassiliki

Papageorgiou, Maria

Papaioannou, Dionissios

Papetti, Adele

Papini, Alessio

Paré, Paul W.

Park, Chung Gyoo

Park, Hee-juhn

Park, Il-Kwon

Park, Jeong Ho

Park, Ji-yeon

Park, Jong Kuk

Park, Jong-Seok

Park, Joo-in

Park, Nam-Gyu

Park, Seung-Chun

Park, Soo-Jin

Park, Wansu

Park, Yong Seek

Parkinson, Don-Roger

Parmeggiani, Fabio

Parolini, Cinzia

Parpinello, Giuseppina Paola

Parsons, J. G.

Parvez, Mohammad
Paschalidis, Konstantinos A.

Pascual, Alberto

Pasinetti, Giulio

Pasini, Dario

Pasini, Luigi

Pasqua, Gabriella

Pasqua, Teresa

Passalaqua, Karla

Passamonti, Sabina

Passarella, Daniele

Passey, Samantha L.

Pastor, Joaquin

Pastoriza, Isabel

Patel, Hardikkumar

Pathuri, Gopal

Patruczynik, Anna

Pauer, Werner

Paulsen, Berit Smestad

Paululat, Thomas

Pautz, Andrea

Pavagadhi, Shruti

Pavic, Valentina

Pavlidis, Ioannis

Pavon-Djavid, Graciela

Pavone, Luigi Michele

Peana, Massimiliano

Peczuh, Mark

Pedatella, Silvana

Pedretti, Alessandro

Pedro, Maireles-Torres

Pélinski, Lydie

Pellecchia, Maurizio

Pellis, Alessandro

Peltonen, Leena

Pelus, Louis

Peluso, Gianfranco

Penczek, Stanislaw

Peneva, Kalina

Penfold, Christopher

Peng, Jinyong

Peng, Robert

Peng, Ruogu

Peng, Wen-Huang 


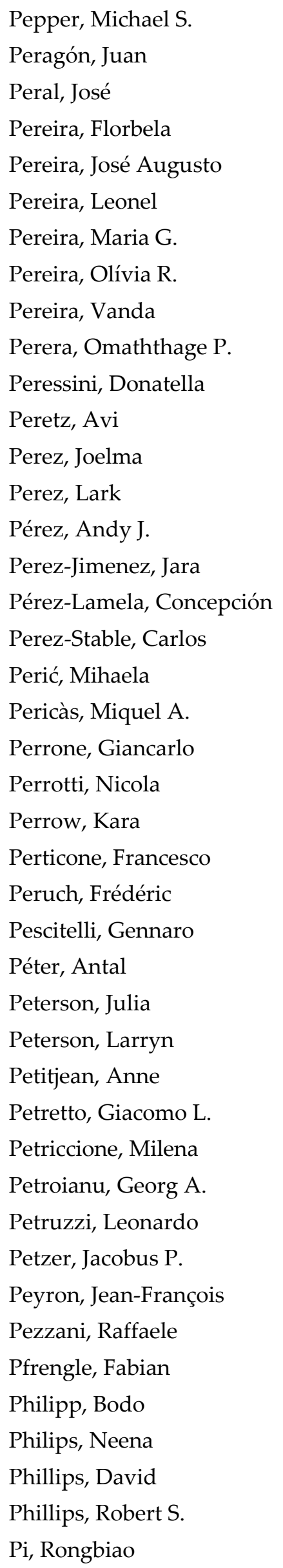

Picariello, Gianluca

Pichler, Martin

Picone, Gianfranco

Piersanti, Giovanni

Pieters, Luc

Pietrucci, Fabio

Pigge, F. Christopher

Pignitter, Marc

Pillay, Viness

Pina, Cristina Della

Pinal, Rodolfo

Pineda, Teresa

Piñeiro Gomez, Marta

Pino-Figueroa, Alejandro

Piñol, Milagros

Pinto, Diana C. G. A.

Pinto, Joana Isabel Monteiro

Piosik, Jacek

Pires, E.

Pires, João

Piro, Benoit

Pisabarro, Antonio G.

Pischetsrieder, Monika

Pittelkow, Michael

Pitucha, Monika

Piva, Terrence

Piwowar, Agnieszka

Piwowarski, Jakub P.

Pizzeghello, Diego

Pizzimenti, Stefania

Planas, Marta

Plano, Daniel

Pla-Quintana, Anna

Plourde, Guy

Pniewski, Tomasz

Poddel'sky, Andrey I.

Pogoń, Krystyna

Pohl, Pawel

Poirier, Raymond A.

Pojman, John A.

Polavarapu, Lakshminarayana

Poleszak, Ewa

Polito, Letizia
Pollier, Jacob

Polyak, Steven

Poma, Paola

Pomerantz, William C. K.

Pons, Antoni

Pons, Josefina

Pontiki, Eleni

Popat, Amirali

Popa-Wagner, Aurel

Popiołek, Łukasz

Popov, Anatoliy

Pöppler, Ann-Christin

Poriel, Cyril

Porter, John

Potamitis, Constantinos

Potapov, Andrei

Potterat, Olivier

Poulsen, Thomas B.

Poupon, Erwan

Poussard, Loïc

Pozsgai, Gabor

Prado, Soizic

Pranantyo, Dicky

Prante, Olaf

Prasad, Sahdeo

Prat, Maria

Pratsinis, Harris

Preti, Delia

Price, Joshua L.

Prieto, Maria

Prieto Garcia, Jose M.

Proestos, Charalampos

Prof. Dr. Martens, Jürgen

Prohens, Rafel

Proschak, Ewgenij

Pruvost, Alain

Psarra, Anna-maria G.

Psomas, George

Ptaszek, Anna

$\mathrm{Pu}$, Ying-Chih

Pucci, Andrea

Puddu, Paolo Emilio

Puglisi, Alessandra 


\begin{tabular}{|c|}
\hline Pulati, Nuerxida \\
\hline Pum, Dietmar \\
\hline Punchard, Neville A \\
\hline Punta, Carlo \\
\hline Pütsep, Katrin \\
\hline Qian, Bing-Jun \\
\hline Qian, Mingxing \\
\hline Qin, Bolin \\
\hline Qin, Wenxin \\
\hline Qiu, Deyou \\
\hline Qiu, Hongdeng \\
\hline Qiu, Ming-hua \\
\hline Quadrelli, Paolo \\
\hline Quan, Guoxing \\
\hline Quave, Cassandra \\
\hline Queiroz, Maria-João R. P. \\
\hline Queiroz Monteiro, Robson \\
\hline Quesenberry, Peter J. \\
\hline Quignard, Françoise \\
\hline Quina, Frank \\
\hline Quintard, Jean-Paul \\
\hline Quintavalla, Arianna \\
\hline Quirino, Rafael \\
\hline Qvit, Nir \\
\hline Rabanal, Francesc \\
\hline Race, Paul \\
\hline Rachek, Lyudmila \\
\hline Rachwalski, Michał \\
\hline Radacsi, Norbert \\
\hline Rades, Thomas \\
\hline Radić, Gordana \\
\hline Rádis-Baptista, Gandhi \\
\hline Radons, Jurgen \\
\hline Raes, Katleen \\
\hline Rafael Ruiz, Jose' \\
\hline Raffa, Demetrio \\
\hline Raffaele, Frazzi \\
\hline Ragauskas, Art J. \\
\hline Ragno, Rino \\
\hline Rahimi, Farid \\
\hline Raj, Madhwa H.G. \\
\hline Rajam, Gowrisankar \\
\hline Rajaputra, Pallavi \\
\hline
\end{tabular}

Rakitin, Oleg

Rakotondraibe, L.H.

Rallis, Michail

Ramalho, João P. Prates

Ramalho, Teodorico

Ramalingam, Latha

Ramidi, Punnamchandar

Ramos, Sonia

Ramos-Ortiz, Gabriel

Ramozzi, Romain

Ramsay, Rona

Ramsden, Lawrence

Ran, Chongzhao

Randall, Stephen K.

Rangnekar, Abhijit

Rao, Shasha

Raposo, Maria Filomena Jesus

Rapposelli, Simona

Rashidinejad, Ali

Rasmuson, Åke

Rasmussen, Helge

Rateb, Mostafa E.

Ratovitski, Edward

Ratz-Łyko, Anna

Rautio, Jarkko

Ravelli, Davide

Rawel, Harshadrai

Ray, Dr Sudip

Ray, Udayan

Raynal, Matthieu

Rebroš, Martin

Reddy, Narsa M.

Reese, R. Neil

Rega, Nadia

Rehrig, Erin M.

Reiger, Matthias

Reis, Catarina Pinto

Reiser, Oliver

Reisman, Scott A.

Rekka, Eleni A.

Remião, Fernando

Remko, Milan

Ren, Dacheng
Ren, Jianguo

Ren, Jun-Li

Renaudineau, Yves

Rescifina, Antonio

Resende Maldonado, Rafael

Revilla, Eugenio

Reyes, Fernando

Reynisson, Jóhannes

Rhee, Young Ho

Rho, Jung-Rae

Ricelli, Alessandra

Richardson, Alan

Riela, Serena

Riemann, Max

Riesen, Hans

Rigano, Daniela

Rijo, Patrícia

Rimondini, Lia

Ringe, Jochen

Rinner, Uwe

Río Segade, Susana

Rioual, S.

Riu-Aumatell, Montserrat

Riva, Renata

Rizzi, Federica

RIzzo, Angela Maria

Rizzuti, Bruno

Robert, Jean-Michel

Robert, Mitchell

Roberto, Dominique

Roberts, Sue A.

Robertson, Mark J.

Robien, Wolfgang

Robledo, Sara M.

Roca-Sanjuán, Daniel

Rocco, Anna

Rochais, Christophe

Roche, Manon

Rodionov, Roman

Rodrigues, Rafael

Rodriguez, Jose

Rodríguez, Jaime

Rodríguez, Mario 


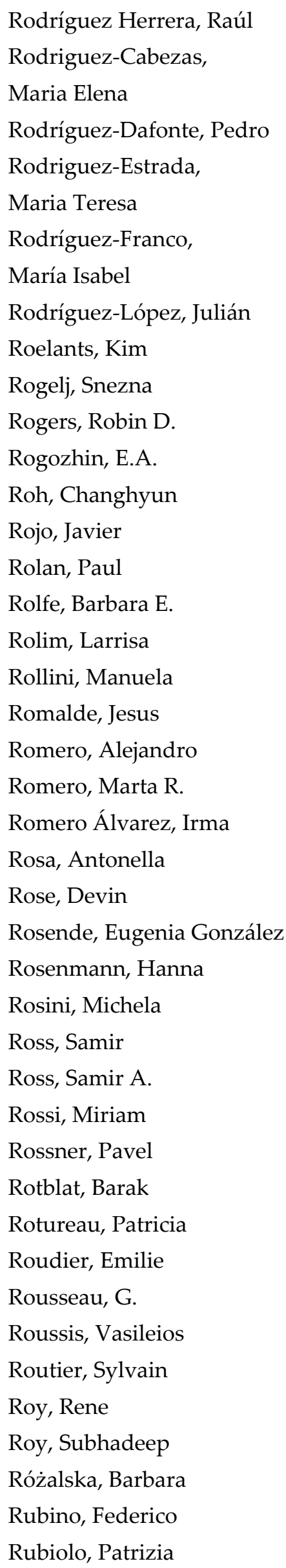

Rucker, Robert B.

Rudick, Jonathan

Rudkowska, Iwona

Rudyk, Olena

Ruelland, Eric

Ruggerone, Paolo

Rühl, Martin

Ruiz, José

Ruiz, T.

Ruiz-Aceituno, Laura

Ruiz-Gómez, Gloria

Rulisek, Lubomir

Ruparel, Shivani

Russo, Maria

Russo, Marina

Ruthstein, Sharon

Rutjes, Floris P. J. T.

Ruzicka, Ales

Ryan, James G.

Ryffel, Bernhard

Ryu, Jong Hoon

Ryu, Mi Heon

S. M., Malathy Sony

S. Silva, M. Luísa

Sabatier, Jean-Marc

Sabila, Paul

Sacks, Gavin

Sackus, Algirdas

Sacré, Pierre-Yves

Saczewski, Jarosław

Sada, Kazuki

Sadiq, Muhammad Waqas

Sagebiel, John C.

Sah, Hong-Kee

Saha, Mrinmoy

Sai Hanuman Sagar, Boddu

Saiano, Filippo

Said, Ahmed M.

Saisho, Yoshifumi

Saito, Shinichi

Sakakibara, Hiroyuki

Sakamoto, Toshio

Sakilam, Satish
Sala, Roberto

Saladino, Raffaele

Salafranca Lázaro, Francisco J.

Salahub, Dennis

Salazar-Olivo, Luis

Saleem, Mohammad

Saleh, Mahmoud A.

Salehi-Reyhani, Ali

Salgo, Professor Andras

Saliba, Anthony J.

Salim, Vonny

Salvatore, Ralph Nicholas

Salvio, Riccardo

Samanidou, Victoria

Samavedi, Satyavrata

Sampson, Clare

Sampson, Nicole S.

San Martin, Carmen

Sanches-Silva, Ana

Sanchez, Christelle

Sanchez, Goar

Sánchez, Gloria

Sánchez Migallón, Ana

Sánchez-García, David

Sánchez-Mata,

María De Cortes

Sánchez-Moreno, Manuel

Sánchez-Pernaute, Olga

Sancho, Teresa

Sandhu, Jagdeep K.

Sandjo, Louis

Sanejouand, Yves-Henri

Sangaraju, Dewakar

Sangireddy, Sasikiran Reddy

Sanjust, Enrico

Sansone, Anna

Sansone, Clementina

Santagati, Andrea

Santangelo, Carmela

Santi, Claudio

Santilio, Angela

Santillo, Michael

Santos, A. Gil 
Santos, Abel

Santos, Clementina

Santos, Roseane

Santos, Valentín

Santos, Willy Glen

Santulli, Gaetano

Sarabia, Francisco

Sarandeses, Luis A.

Sarazin, Catherine

Sarkar, Biprajit

Sartillo-Piscil, Fernando

Satake, Honoo

Šatínský, Dalibor

Sato, Naoyuki

Sato, Soshi

Sato, Sota

Satora, Pawel

Saturnino, Carmela

Satyal, Prabodh

Saulnier, Luc

Savoia, Dianella

Sayas, Estrella

Sbardella, Gianluca

Scala, Angela

Scalenghe, Riccardo

Scarfato, Paola

Scarso, Alessandro

Schafleitner, Roland

Schaich, Karen

Schaller, Hubert

Schepdael, Ann Van

Schinkovitz, Andreas

Schirhagl, Romana

Schmeda-Hirschmann,

Guillermo

Schmid, J.

Schmid, Matthias

Schmiderer, Corinna

Schmidl, Doreen

Schmidt, Bernd

Schmidt, Martin

Schmidt, Susann

Schmidtke, Michaela
Schmitz, John C.

SchneeklothJr., John S.

Schneider, Bernd

Schneider, Raphael

Schnermann, Martin J.

Scholz, Markus

Scholz-Ahrens, Katharina

Schonbrun, Ethan

Schönherr, Holger

Schörken, Ulrich

Schrag, Matthew

Schroeder, Grzegorz

Schuck, Alyssa

Schulz, Benjamin L.

Schulz, Frank

Schulzová, Věra

Schurig, Volker

Schuurhuis, Gerrit J.

Schwartz, Stanley A.

Schwendicke, Falk

Scognamiglio, Monica

Scorziello, Antonella

Scotti, Marcus

Seabra, Amedea B.

Seca, Ana

Seca, Ana M. L.

Seebacher, Werner

Seebeck, Florian P

Segarra-Marti, Javier

Seger, Christoph

Segi, Masahito

Ségui, Bruno

Seifert, Karlheinz

Seijas Vázquez, Julio

Sekine, KenTaro

Seley Radtke, Kathie

Selli, Elena

Selma, María V.

Sémeril, David

Sen, Arundhuti

Sen, Sanghamitra

Sendker, Jandirk

Seo, Jae Hong
Seo, Seung-Yong

Seo, Woo Duck

Seo, Yongbeom

Seo, Young-Soo

Seol, Geun Hee

Séraphin, Denis

Sergi, Consolato

Serio, Annalisa

Serpone, Nick

Serra, Angels

Serra, Immacolata

Serra, Raffaele

Serra, Stefano

Serralheiro, Maria Luísa M.

Serrano, Jose C. E.

Serrano, Maria

Servin, Alain L.

Sestili, Piero

Seth, Devanshi

Sethi, Gautam

Setzer, William

Sgarbossa, Paolo

Sgarra, Riccardo

Sgorbini, Barbara

Shah, Dhaval K.

Shah, Dilip

Shahar Yar, Mohammad

Shahbazi, Mohammad Ali

Shaio, Young-ji

Shalin, Sara

Shangguang, Dihua

Shankar, Eswar

Shapiro, Bruce A.

Sharma, Anjali

Sharma, Arun K

Sharma, Bhesh

Sharma, Sherven

Shaw, Anthony

Shaw, Pang-Chui

Shaw, Subrata

Shehu, Amarda

Sheikh, Farah

Sheldon, Julie 
Shen, Jun

Shen, Qiang

Shen, Tang-Long

Shen, Xu Shen

Shen, Ya-Ching

Shen, Yue-mao

Sheng, Rong

Sherwood, James

Sheu, Joen-Rong

Shi, Daaing

Shi, Dayong

Shi, Hua

Shi, Jie-hua

Shi, Qiang

Shiau, Chung-Wai

Shieh, Chwen-Jen

Shih, Wen-Ling

Shiina, Isamu

Shima, Seigo

Shimada, Shigeru

Shimizu, Yohei

Shimoda, Hiroshi

Shimodaira, Shigetaka

Shimoi, Norihiro

Shimomura, Norihiro

Shin, Beom Soo

Shin, Chae-Ho

Shin, Dong-Soo

Shin, Injae

Shin, Jae Sup

Shinada, Tetsuro

Shinn, Sara

Shinomiya, Kazufusa

Shipley, Paul

Shiragami, Tsutomu

Shirai, Keiko

Shirakami, Yohei

Shirakawa, Hitoshi

Shirasawa, Kenta

Shiro, Tomoya

Shirsath, Sagar

Shityakov, Sergey

Shrestha, Gajendra
Shukla, Shivendra D.

Siggelkow, Heide

Sikorska, Hanna

Siligardi, Giuliano

Silipo, Alba

Silks, Louis A.

Silva, Ana C

Silva, Ana M.g.

Silva, Anjana

Silva, Branca M.

Silva, Daniel Varon

Silva, Filomena

Silva, Jorge Carvalho

Silva, Luís

Silva, Luís R.

Silva, Maria Fátima

Silva, Maria Fernanda

Silva, Sónia

Silvar, Cristina

Silvério, Sara C.

Silverman, Richard B.

Silvestri, Elena

Silvestri, Romano

Simanek, Eric

Simes, Dina

Simirgiotis, Mario J.

Simmons, Katie

Simmons, Peter A.

Simonsen, Henrik Toft

Simpson, Bradley S.

Simpson, Garth J.

Sims, Ian M.

Singer, Robert J.

Singh, Ashok K.

Singh, Ashutosh

Singh, Gurpreet

Singh, Kamalendra

Singh, Nisha

Sinha, Birandra K.

Sinicropi, Adalgisa

Siniscalco, Dario

Siodłak, Dawid

Sirusta, Silvia
Sisto, Margherita

Siwek, Agata

Sjogren, Klara

Skalicka - Woźniak, Krystyna

Skaltsa, Helen D.

Skaltsounis,

Alexios-Leandros

Skardal, Aleksander

Skellam, Elizabeth

Skenderi, Katerina P.

Skovgaard, Ole

Slavik, Roger

Sławiński, Jarosław

Sledz, Pawel

Słoczyńska, Karolina

Smani, Younes

Smart, Laura

Smeds, Annika I.

Smith, Caroline

Smith, Charles J.

Smith, Judith A.

Smith, Robert A.

Smith, Terry

Smoleński, Piotr

Smoliga, James

Sobkowski, Michal

Sobral, Abilio J. F. N.

Socaciu, Carmen

Soengas, Raquel

Soeta, Takahiko

Sohn, Jeongwon

Sohtome, Yoshihiro

Sokolove, Jeremy

Solà Alberich, Rosa

Solano, Francisco

Solfrizzo, Michele

Solinís, M. Ángeles

Solomon, Melani

Soloshonok, Vadim A.

Solsona, Benjamín

Sone, Hideko

Song, Jaewhan

Song, Jinhua 
Song, Juha

Song, Kenan

Song, Kwang Ho

Song, Mei

Song, Sang Hoon

Song, Shao-Jiang

Song, Yang-heon

Sonsalla, Patricia K

Sopade, Peter A.

Sørensen, Jens Laurids

Sorokin, Alexander

Sosa, Silvio

Soto, Carmen

Soukup, Garrett

Soukup, Sebastian T.

Soulère, Laurent

Sousa, Emília

Souza-Smith, Flavia

Spangenberg, Bernd

Spano, Giuseppe

Sparr, Christof

Speck, Peter G.

Spégel, Peter

Spingler, Bernhard

Spiteller, Peter

Spyrakis, Francesca

Sreerama, Subramanya

Sri Vedavyasa Sri, Radhakrishnan Stratakis, Manolis

Sridhar, Jayalakshmi

Srinivas, Keerthi

Srivatsan, Eri S.

Srivatsan, Malathi

Stacchiotti, Alessandra

Stahl, Frank

Staines, K.A.

Stallforth, Pierre

Staneva, Galya

Stanley, Jessica

Stapleton, P. A.

Stavropoulos, George P.

Stavros, Vasilios

Stec, David E.

Stec, Jozef

Struga, Marta
Stecca, Barbara

Stefanidis, Georgios.

Stehle, Jörg H.

Steindel, Mário

Steingass, Christof B.

Stella, Lorenzo

Stenstrøm, Yngve

Stephen, Michael Rajesh

Stevenson, Paul

Stewart, Alan V.

Stewart, Alexandre

Stewart, Cameron R.

Stewart, Teneale A.

Steyn, Frederik

Stiborova, Marie

Sticherling, Michael

Stierle, Andrea

Stine, Keith J.

Stipanovic, Arthur

Stiriba, Salah-Eddine

Stirpe, Fiorenzo

Stockdill, Jennifer L.

Stockland, Robert A.

Stojakowska, Anna

Stone, William

Stournaras, Christos

Strano, Sabrina

Strekowski, Lucjan

Streubel, Rainer

Strube, Jochen

Stuchebrukhov, Alexei

Studt, Lena

Stüger, Harald

$\mathrm{Su}, \mathrm{Chun}-\mathrm{Li}$

$\mathrm{Su}$, Chunming

$\mathrm{Su}$, Huanxing

$\mathrm{Su}$, Jui-Hsin

Su, Xiao Q

$\mathrm{Su}$, Zhiqiang

$\mathrm{Su}, \mathrm{Zi}$-Ren

Subramaniam, Dharmalingam
Subramaniam, Prasad

Sucheck, Steven

Suckling, Colin J.

Suga, Hiroaki

Suga, Seiji

Sugahara, Takuya

Sugai, Takeshi

Sugimoto, Kenji

Sugiura, Yoshimasa

Suh, Hyung Joo

Suib, Steven L.

Suklje, Katja

Sukocheva, Olga

Sulman, Esther

Sun, Dr. Grace

Sun, Guohui

Sun, Jian

Sun, Litao

Sun, Peilong

Sun, Shi

Sun, Wenjie

Sun, Zhonghua

Sunagar, Kartik

Sunatsuki, Yukinari

Sunavala-Dossabhoy,

Gulshan

Sung, Mi Jeong

Sung, Ping-Jyun

Sunseri, Francesco

Surup, Frank

Sutton, Troy

Suzuka, Toshimasa

Svedberg, Marie M.

Sweetman, Crystal

Swindle-Reilly, Katelyn E.

Swinney, David C.

Syed, Viqar

Sykes, Melissa

Szekely, Gyorgy

Szilvási, Tibor

Szöllősi, Daniel

Szpilman, Alex M.

Sztanke, Małgorzata 
Szumny, Antoni

Szunerits, Sabine

Tabanca, Nurhayat

Tabata, Yasuhiko

Tachibana, Shinjiro

Tadić, Vanja

Taherzadeh, Mohammad

Tahir, Prof. Nawaz

Tai, Cheng-Jeng

Taillefumier, C.

Taira, Junsei

Tajima, Takahisa

Tak, Jun-Hyung

Takagaki, Atsushi

Takahama, Umeo

Takahashi, Daisuke

Takanami, Toshikatsu

Takano, Katsura

Takebe, Naoko

Takei, Takashi

Takenaka, Shinji

Takeshita, Fumitaka

Takita, Ryo

Takizawa, Shinobu

Talbert, Joey N.

Talele, Tanaji $\mathrm{T}$

Tam, Kin

Tamanoi, Fuyuhiko

Tamassia, Nicola

Tame, Jeremy

Tamura, Osamu

Tan, Cai-Ping

Tan, Dunxian

Tan, Jianwen

Tanahashi, Takao

Tanaka, Aparecida Sadae

Tanaka, Naoki

Tanaka, Shigenori

Tanaka, Takayuki

Tang, You-Zhi

Tang, Yu

Tang, Yu-Ping

Tanida, Mamoru
Taniguchi, Nobukazu

Tanimoto, Hiroki

Tantillo, Dean

Tao, Yuanqi

Tappia, Paramjit S.

Tarantino, Giovanni

Tarasova, Nadya I.

Tarkowska, Danuse

Tarr, D Ellen

Tava, Aldo

Tavano, Lorena

Tavares Carvalho, José Carlos

Tavazzi, Barbara

Tavío, Maria Del Mar

Tawata, Shinkichi

Taylor, James E.

Tempesta, Maria

Terán, Carmen

Terner, James

Terzyk, Artur P.

Teschke, Rolf

Tessore, Francesca

Teufel, Robin

Tewari-Singh, Neera

Tezuka, Yasuhiro

Thakur, Chitra

Thamattoor, Dasan M.

Thiéry, Valérie

Thiriet, Marc

Thirunavukkarasu, Mahesh

Thomas, Eric

Thomas, Sajesh P.

Thomas, Samuel

Thomas, Shane

Thomas, Stephen P.

Thomas, T. J.

Thomas, Walter G.

Thöming, Gunda

Thomsen, Allan Randrup

Thoo-Lin, Paul Kong

Thorwart, Michael

Thota, Sreekanth

Thounaojam, Menaka
Thunders, Michelle

Tidwell, Thomas

Tietjen, Ian

Timperley, Christopher M.

Ting, Richard

Ting, Simon

Tiritan, Maria Elizabeth

Titinchi, Salam

Titorenko, Vladimir I.

Tiziano, Tuccinardi

Tognetti, Vincent

Tojo, Emilia

Tokuyama, Shogo

Tolosa, Josefa

Tomalia, Donald

Tomé, Augusto C.

Tomi, Félix

Tomicic, Maja

Tomitaka, Asahi

Tommasi, Sara

Tommonaro, Giuseppina

Tomoda, Hiroshi

Tomofuji, Takaaki

Tonelli, Michele

Toohey, John

Tooyama, Ikuo

Top, Siden

Tormo, José Rubén

Török, Marianna

Toropov, Andrey A.

Torrens, Francisco

Torrents, Eduard

Torricelli, Renzo

Touchard, Axel

Toy, Randall

Toydemir, Gamze

Toyoda, Atsushi

Traba, C.

Trabace, Luigia

Trabolsi, Ali

Tresini, Maria

Trincone, Antonio

Trindade, 
Reginaldo Almeida da

Tringali, Corrado

Tripathi, Siddharth Kaushal

Tripodi, Gianluca

Tripp, Carl P.

Trippier, Paul C.

Trivedi, Drupad K.

Trombetta, Domenico

Trombini, Claudio

Truchado, Pilar

Trujillo Rodríguez, María José

Truong Phuoc, Nghia

Trzeciak, Anna

Tsai, Cheng-Fang

Tsai, Chia-Fang

Tsai, Chia-Wen

Tsai, Fuu-Jen

Tsai, I-Lin

Tsai, Sheryl

Tsai, Tsung-Yu

Tsang, Siu Wai

Tsantili-Kakoulidou, Anna

Tsao, Chia-Wen

Tsay, Hien-Yie

Tseng, Chih-Hua

Tseng, Wei-Lung

Tsim, Karl

Tsipis, Athanassios C.

Tsoleridis, Constantinos A.

Tsopmo, Apollinaire

Tsotsis, Theodore T.

Tsoungas, Petros G.

Tsugawa, Hiroshi

Tsui, Stephen Kwok-Wing

Tsuji, Hayato

Tsuji, Yasuhide

Tsukamoto, Masaki

Tsukiyama-Kohara, Kyoko

Tsurkan, Mikhail V.

Tsutsui, Makusu

Tuão Gava, Carlos Alberto

Tuccinardi, Tiziano

Tufarelli, Vincenzo
Tulkens, Paul

Tulla Puche, Judit

Tullius Scotti, Marcus

Tung, Bui Thanh

Tuononen, Heikki M.

Turrini, Eleonora

Tyagi, Pradeep

Tyler, Betty

Tyndall, Joel

Tzakos, Andreas G.

Ubiali, Daniela

Ubukata, Takashi

Uccello-Barretta, Gloria

Uddin, Zakir

Uehara, Tomoya

Ueno, Takafumi

Uji-I, Hiroshi

Ukic, Sime

Ulatowski, Lynn

Ulisse, Salvatore

Ullah, Aman

Ulrich, Craig

Ulrich, Joachim

Uno, Tomohide

Unrau, Peter

Urbani, Maxence

Urbanska, Ewa M.

Urbieta, María Tabernero

Urra, José Miguel

Usami, Yoshihide

Usui, Kazuteru

Usui, Kenji

Usui, Takeo

Usuki, Yoshinosuke

Utsumi, Shigenori

Vachali, Preejith

Vajda, Sandor

Vakkilainen, Esa

Valcheva-Kuzmanova, Stefka

Valdes, Esperanza

Vale, Nuno

Vale, Paulo

Valencia, Gregorio
Valentao, Patrícia

Valente, Sergio

Valentova, Katerina

Valero, Manuel

Valgimigli, Luca

Valiyaveettil, Suresh

Valles, Soraya L.

Van Calenbergh, Serge

van den Broek, Lambertus

Van Den Ende, Wim

Van Der Stelt, Mario

Van Dyke, Knox

Van Ginkel, Paul

Van Griensven, Leo J. L. D

Van Lanen, Steven

Van Tassel, Paul

Van Waardenburg,

Robert C. A. M.

Vanamala, Jairam K. P.

Vanderlei, Maria de Fátima

Vanelle, Patrice

Vangala, Venu

Vankayala, Raviraj

Varani, Luca

Varela-López, Alfonso

Varela-Ramirez, Armando

Varesano, Alessio

Vargas, Rubem M. F.

Varvounis, George

Vasilyev, Aleksander

Vaughan, Roger A.

Vávrová, Kateřina

Vavvas, Demetrios G.

Vazquez-Martínez,

José Alfredo

Vegvari, Akos

Veiga Jr, Valdir Florencio

Veleeparambil, Manoj

Velez, Zélia

Velkov, Tony

Venditti, Alessandro

Vennerstrom, Jonathan

Venus, Joachim 
Vepsäläinen, Jouko

Vergadi, Eleni

Vergara, Daniele

Verma, Deeptak

Verma, Madan

Verma, Ranjit

Verma, Sanny

Vernekar, Sanjeev Kumar

Verri Jr., Waldiceu A.

Vertuani, Silvia

Vervoort, Jacques

Vetter, Stefan W.

Vetvicka, Vaclav

Viapiana, Agnieszka

Vidal, Joëlle

Videira, Arnaldo

Vieira, Mónica

Vignolle, Joan

Vilanova, Manuel

Vilanova, Neus

Vilela, Alice

Villalobos, Carlos

Villalonga, Reynaldo

Villani, Claudio

Villemagne, Victor L.

Villoutreix, Bruno

Vinale, Francesco

Viñas, Miguel

Vincenzo, Lattanzio

Vinogradov, Evguenii

Virjamo, Virpi

Visa, Maria

Viso, Alma

Vistoli, Giulio

Vitalini, Sara

Vitillo, Jenny G.

Vitorino, Rui

Vittadini, Andrea

Viuda-Martos, Manuel

Vivo, Paola

Vlase, Laurian

Vo, Minh D.

Volety, Aswani K.
Volpe, Maria

Von Der Mark, Klaus

Von Wright, Atte

Vosteen, Ilka

Vougas, Kostas

Vougogiannopoulou,

Konstantina

Voutquenne, Laurence

$\mathrm{Vu}$, Chi B.

Vuong, Quan V.

Vuong, Quan Van

Wada, Yuji

Wadsak, Wolfgang

Wagner, Anika

Wagner, Gabriele

Wahab, M. Farooq

Waheed, Abdul

Wahyudi, Hendra

Wakabayashi, Hiroki

Waksmundzka-Hajnos,

Monika

Waldmeier, Felix

Walker, Jessica

Walker III, William B.

Wallis, Russell

Walls, Zac

Walsby, Charles J.

Walser, Tonya C.

Waltenberger, Birgit

Wan, Jianbo

Wan, Xiao-Chun

Wan, Xuehua

Wang, Chang-hong

Wang, Chao-Min

Wang, Chi Chiu

Wang, Chin-Kun

Wang, Chrong-Reen

Wang, Cunde

Wang, David

Wang, DongDong

Wang, Gou-Jen

Wang, Haiqiao

Wang, I-Jong
Wang, Jeffrey

Wang, Jeh-jeng

Wang, Jin

Wang, Junjian

Wang, Lili

Wang, Liping

Wang, Michael

Wang, Peng

Wang, Piwen

Wang, Quan

Wang, Qun

Wang, Robert Y. -L.

Wang, Sanwu

Wang, Sheng-yang

Wang, Wei

Wang, Wei-Lung

Wang, Wentian

Wang, Xiangke

Wang, Xiao-Bing

Wang, Xiaohong

Wang, Xiaosong

Wang, Xiaowu

Wang, Xin

Wang, Xinkun

Wang, Xin-Luan

Wang, $\mathrm{Xu}$

Wang, Xue-Song

Wang, Yi

Wang, Yifei

Wang, Ying

Wang, Yongheng

Wang, Yun-Ming

Wang, Zhengtao

Wang, Zhiwei

Wang, Zuankai

Wangchuk, Phurpa

Wansi, Jean Duplex

Ward, Sara Jane

Waseem, Raza

Waser, Mario

Wąsik, Tomasz J.

Watanabe, Masatoshi

Watanabe, Ryuichi 
Watanabe, Tatsuo

Watkins, A. Neal

Watkins, G. Leonard

Waxman, Joshua

Weber, John T

Wehmschulte, Rudolf J.

Wei, Da-Hua

Wei, Guor-Jien

Wei, Hui

Wei, Qufu

Wei, Wu

Weinhold, Frank

Weinstein, Julia A.

Weising, Kurt

Welch, Kevin D.

Welker, Mark E.

Welte, Cornelia U.

Wendel, Graciela Haydée

Weng, Alexander

Weng, Ching-Feng

Weng, Duan

Weng, Meng-Shih

Weng, Qunhong

Wertz, Philip W.

Wesolowski, Marek

West, Caroline

West, Robert

Westcott, Stephen A.

Weston, Leslie

Weston, Paul A.

Westwell, Andrew

Westwood, Nicholas J.

White, Mark G.

Whitford, Paul C

Wiczk, Wiesław

Wiczkowski, Wieslaw

Wiesner, Melanie

Wietrzyk, Joanna

Wilking, Janet

Wilkinson, Brendan

Wilkinson, J. M.

Wilkinson, Mark D.

Williams, Gavin
Williams, Noelle S.

Williams, Robert J.

Williams, Vance

Williamson, Mike

Willis, Michael C.

Wilson, Brenda Anne

Wilson, David B.

Winckler, Thomas

Windle, Henry J.

Wink, Michael

Winkler, Johannes

Winter, Heiko

Wirth, Thomas

Wisconsin, Bloodcenter

Witcher, Michael

Witczak, Zbigniew

Witkowski, Stanisław

Woelwer-Rieck, Ursula

Wohl, Christopher J.

Wohlrab, Sebastian

Wojaczyńska, Elżbieta

Wojdyło, Aneta

Wölfle, Ute

Wong, Chung F.

Wong, Fung-Fuh

Wong, Ka-Leung

Wong, Man-Sau

Wong, Sze Choong

Wong, Vincent Kam Wai

Wood, Bayden

Woodman, Owen

Woodward, Robert T.

Woon, Esther

Woźniak, Dorota

Wrenger, Carsten

Wright, Colin W.

$\mathrm{Wu}$, Bing

Wu, Bin-Nan

$\mathrm{Wu}$, Chi-Rei

$\mathrm{Wu}$, Chung-Chih

$\mathrm{Wu}$, Ho-Shing

$\mathrm{Wu}$, Hua

$\mathrm{Wu}$, Huaizhu
$\mathrm{Wu}$, Jianyong

$\mathrm{Wu}$, Jin-Yi

$\mathrm{Wu}$, Li-Chen

$\mathrm{Wu}$, Ming-Jiuan

$\mathrm{Wu}$, Ming-Jung

Wu, Nick

$\mathrm{Wu}, \mathrm{Pao}-\mathrm{Chu}$

Wu, Quan-Xiang

$\mathrm{Wu}$, Sheng-Chi

Wu, Shih-Hsiung

$\mathrm{Wu}$, Shihua

$\mathrm{Wu}$, Shin-Tson

Wu, Tung-Kung

Wu, Tzong-Yuan

$\mathrm{Wu}$, Wen-Huey

$\mathrm{Wu}$, Xiaofeng

Wu, Xiaoyu

Wu, Yang-Chang

$\mathrm{Wu}$, Zhenghong

$\mathrm{Wu}$, Zhenqiang

$\mathrm{Wu}$, Zhisheng

$\mathrm{Wu}$, Alan $\mathrm{Hb}$

Wulff, Jeremy E.

Wyman, Ian

Xavier, Nuno Manuel

Xi, Guifa

Xi, Weixian

Xia, Weiming

Xie, Chong

Xie, Guoxiang

Xie, Jinghang

Xie, Liming

Xie, Qian

Xie, Ying

Ximing, Wang

Xin, Dongyue

Xing, Junji

Xiong, Yan

$\mathrm{Xu}$, Baoshan

$\mathrm{Xu}$, Jie

$\mathrm{Xu}$, Peisheng

$\mathrm{Xu}$, Qiongming

$\mathrm{Xu}$, Wang 


Xu, Xingshun
Xu, Xudong
Xu, Xueming
Xu, Yan
Xu, Yi Jun
Xu, Yun
Xuan, Tran Dang
Yaghmur, Anan
Yahia, L'Hocine
Yajima, Shunsuke
Yamada, Kiyofumi
Yamada, Sohsuke
Yamada, Yusuke
Yamagishi, Takehiro
Yamaguchi, Kanji
Yamaguchi, Masahiko
Yamaguchi, Yoshihiro
Yamaki, Kohji
Yamaki, Kouya
Yamamoto, Yasunori
Yamanaka, T.
Yamashita, Teruhito
Yang, Qianru
Yamauchi, Satoshi
Yan, Liu, Minsong
Yamazaki, Hiroyuki
Yan, Dongqing
Yan, Hong
Yan, Xuehai
Yan Long, Gu
Yanagita, Ryo C.
Yanase, Emiko
Yang, Cheng
Yang, Chia-Ning
Yang, Chi-Chiang
Yang, Ding-I
Yang, Ding-Yah
Yang, Guangdong
Yang-Ching
Yang-Wei
Yang, Hong

Yang, Seung Ok
Yang, Si Kyung
Yang, Wei-Dong
Yang, Wei-Hsiung
Yang, Wen-Bin
Yang, Xiuwei
Yang, Yao
Yang, Ya-Tang
Yang, Yu-Chiao
Yang, Yu-Liang
Yang, Zhen
Yang, Zhijun
Yao, Ching Fa
Yao, Zhili
Yao, Zhong-Ke
Yaoita, Yasunori
Yarmolinsky, Ludmila
Yasukawa, Kiyoshi
Yau, Sonata
Ye, Deyong
Ye, Zhaoyang
Ye, Zhibin
Yedery, Roshan D.
Yeh, Chih-ko
Yeh, Jan-ying
Yeh, Ming-Chang P.
Yeh, Pamela
Yeh, Shu-Lan
Yen, Chia-Hung
Yen, Feng-Lin
Yeong, Wai Yee
Yoko, Jung Sun
Yokobolu, Jayasudhan Reddy Kai E. O.
Yiannakopoulou, Eugenia
Yim, Changyong
Yin, Guowei
Yin, Mei-chin
Yin, Sheng
Yin, Zhiwei
Ylijutomu
Yooihito
Yong

Yoon, Do-Young

Yoon, In-Soo

Yoon, Kee Dong

Yoon, Moon-Young

Yoon, Tae-Hoon

Yoshida, Jun

Yoshida, Suguru

Yoshida, Takashi

Yoshihito, Yokoyama

Yoshikawa, Masaaki

Yoshimoto, Makoto

Yoshimura, Yuichi

Yoshioka, Naoki

You, Liang

You, Zhonglu

Young, Jette F.

Young, Sarah

Yu, Chengzhong

$\mathrm{Yu}$, Donghong

Yu, Guangli

Yu, Hua

Yu, Jinhong

Yu, Meihua

Yu, Shi-Shan

$\mathrm{Yu}$, Shouyun

$\mathrm{Yu}$, Wenquan

$\mathrm{Yu}$, Yinghua

Yuan, CS

Yuan, Yaxia

Yuan, Youyong

Yuan, Zhao

Yuba, Eiji

Yue, PatrickYingKit

Yumoto, Hiromichi

Yun, Soon-Il

Yun, Young Sook

Zabetakis, Loannis

Zabotina, Olga

Zaccheria, Federica

Zaferanloo, Bita

Zagotto, Giuseppe

Zakrzewicz, Dariusz

Zakrzewski, Jerzy 


\begin{tabular}{|c|c|c|}
\hline Zamaratskaia, Galia & Zhang, Kun & Zhou, Jiajing \\
\hline Zambrowicz, Aleksandra & Zhang, Liangliang & Zhou, Ligang \\
\hline Zamora, Fernando & Zhang, Longhe & Zhou, Yifa \\
\hline Zanardi, Chiara & Zhang, Qiang & Zhu, Can \\
\hline Zanetti, Michela & Zhang, Qinghai & Zhu, Junwei J. \\
\hline Zannatal, Ferdous & Zhang, Qingwen & Zhu, Prof. Dr. Changjin \\
\hline Zaprutko, Lucjusz & Zhang, Shaozhong & Zhu, Xingyuan \\
\hline Zard, Samir Z. & Zhang, Shu & Zidorn, C \\
\hline Zarrelli, Armando & Zhang, Wei & Ziegler, Thomas \\
\hline Zarubaev, Vladimir & Zhang, Xiquan & Zielińska, Sylwia \\
\hline Zawilska, Jolanta B. & Zhang, Yanmin & Zielińska-Pisklak, Monika A. \\
\hline Zehnacker-Rentien, Anne & Zhang, Yixiang & Zielonka, Jacek \\
\hline Zeiner, Michaela & Zhang, Yonghong & Ziemba, Barbara \\
\hline Zeiner, Tim & Zhang, Yuhang & Zimmer, Reinhold \\
\hline Zeller, Wayne E. & Zhang, Yuqing & Ziogas, Dimosthenis \\
\hline Zeng, Dexing & Zhang, Z. & Ziółek, Marcin \\
\hline Zeng, Huaqiang & Zhang, Zhanhui & Zi-Sheng, Luo \\
\hline Zeng, Jie & Zhang, Zhiping & Zitko, Jan \\
\hline Zeng, Wenbin & Zhang, Zhuo & Złotek, Urszula \\
\hline Zeng, Xiaoming & Zhao, Hongbo & Żołnowska, Beata \\
\hline Zerbetto, Francesco & Zhao, Jincai & Zong, Xu-xiao \\
\hline Zgoła-Grześkowiak, Agnieszka & Zhao, Jin-Hao & Zoranovic, Tamara \\
\hline Zhang, Chi & Zhao, Liang & Zordoky, Beshay \\
\hline Zhang, Dawei & Zhao, Yan & Zotchev, Sergey \\
\hline Zhang, Dengsong & Zhao, Yanchuan & Zou, Taotao \\
\hline Zhang, Fan & Zheng, Gaowei & Zoumpoulakis, Panagiotis \\
\hline Zhang, Ge & Zheng, Qi-Huang & Zrenner, Rita Maria \\
\hline Zhang, Guojian & Zheng, Shilong & Zrnčić, Mirta \\
\hline Zhang, Guolin & Zheng, Weijie & Zuo, Li \\
\hline Zhang, Hong & Zheng, Weijun & Zupko, Istvan \\
\hline Zhang, JingJing & Zheng, Ying & Zygmunt, Małgorzata \\
\hline Zhang, Jinsong & Zhong, Guohua & Zylinska, Ludmila \\
\hline Zhang, Junbo & Zhong, Haizhen & \\
\hline Zhang, Junying & Zhong, Tuhua & \\
\hline Zhang, Junzeng & Zhou, Cong-Ying & \\
\hline Zhang, Kai & Zhou, Hai-Bing & \\
\hline
\end{tabular}

() 2017 by the author; licensee MDPI, Basel, Switzerland. This article is an open access article distributed under the terms and conditions of the Creative Commons Attribution (CC-BY) license (http://creativecommons.org/licenses/by/4.0/). 